\title{
Numerical simulation of rivulet evolution on a horizontal cable subject to an external aerodynamic field
}

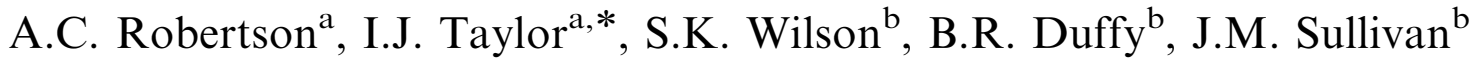 \\ ${ }^{a}$ Department of Mechanical Engineering, University of Strathclyde, Glasgow G1 1XJ, UK \\ ${ }^{\mathrm{b}}$ Department of Mathematics, University of Strathclyde, Glasgow G1 $1 X H, U K$
}

Received 17 July 2008; accepted 10 September 2009

Available online 7 November 2009

\begin{abstract}
On wet and windy days, the inclined cables of cable-stayed bridges may experience a large amplitude oscillation known as rain-wind-induced vibration (RWIV). It has previously been shown by in situ and wind-tunnel studies that the formation of rain-water accumulations or 'rivulets' at approximately the separation points of the external aerodynamic flow field and the resulting effect that these rivulets have on this field may be one of the primary mechanisms for RWIV. A numerical method has been developed to undertake simulations of certain aspects of RWIV, in particular, rivulet formation and evolution. Specifically a two-dimensional model for the evolution of a thin film of water on the outer surface of a horizontal circular cylinder subject to the pressure and shear forces that result from the external flow field is presented. Numerical simulations of the resulting evolution equation using a bespoke pseudo-spectral solver capture the formation of two-dimensional rivulets, the geometry, location and growth rate of which are all in good agreement with previous studies. Examinations of how the distribution and magnitude of aerodynamic loading and the Reynolds number influence the rivulet temporal evolution are undertaken, the results of which indicate that while all three affect the temporal evolution, the distribution of the loading has the greatest effect.
\end{abstract}

(C) 2009 Elsevier Ltd. All rights reserved.

Keywords: Rain-wind-induced vibration; Circular cylinder; Numerical simulation; Pseudo-spectral method; Thin-film approximation

\section{Introduction}

On wet and windy days, the inclined cables of cable-stayed bridges may experience a large amplitude oscillation known as rain-wind-induced vibration (RWIV). Such oscillations have been recorded on several bridges worldwide since the phenomenon was initially observed on the Meikonishi Bridge near Nagoya, Japan, as reported by Hikami and Shiraishi (1988). Notable amongst the literature are the Erasmus Bridge, Rotterdam, The Netherlands, (Geurts et al., 1998), the Fred Hartmann Bridge, Baytown, Texas (Zuo et al., 2008), and the Dongting Lake Bridge, Hunan Province, China (Ni et al., 2007), where full scale in situ investigations have been undertaken. Such studies have led to a sizeable knowledge base being generated as to the conditions under which RWIV most commonly occurs.

\footnotetext{
*Corresponding author. Tel.: + 44 1415483753; fax: + 441415525105 .

E-mail address: ian.taylor@strath.ac.uk (I.J. Taylor).
} 
In summary, this data suggests that RWIV tends to occur only over a restricted range of wind velocities (typically $5-15 \mathrm{~m} / \mathrm{s}$ ), under moderate rain conditions, for cables that descend in the direction of the wind. The response itself normally occurs in the cable-pylon plane with a larger amplitude and lower frequency than vortex-induced vibration. Through such characteristics RWIV can thus be identified as a distinct aeroelastic phenomenon, with particular features that distinguish it from other aeroelastic instabilities such as galloping or the aforementioned vortex-induced vibration.

It has previously been reported that in cases where RWIV does occur, the thin film of rain water present on the cable normally accumulates to form two rivulets near the separation points of the external aerodynamic field around a dry cable (Bosdogianni and Olivari, 1996). Although in practice rivulets are a three-dimensional phenomenon with axial fluid flow, herein the term rivulet will also be used to represent the accumulations of fluid in the present twodimensional solution. The experimental wind-tunnel studies undertaken to support the full-scale tests, such as those of Bosdogianni and Olivari (1996), Matsumoto et al. (1995), Flamand (1995), Verwiebe and Ruscheweyh (1998), Cosentino et al. (2003), Gu and Du (2005) and Wang et al. (2005), use rivulets of varying origin (some using 'artificial' rivulets, but others allowing rivulets to form 'naturally' from a film of water), but all the above authors accept that a rivulet must be present for RWIV to occur, and so any numerical simulation of the RWIV instability should also include the formation and evolution of these rivulets. Likewise several analytical models such as those of Yamaguchi (1990), Gu and Huang (2008) and Peil and Dreyer (2007) have also been developed, and while these display individual nuances determined by the exact nature of the particular aspect under investigation, they typically use a twodimensional, multiple mass, multiple degree-of-freedom, spring mass damper system and a quasi-steady approximation of the aerodynamic forces to represent the RWIV phenomenon.

In comparison to experimental and analytical models, however, computational models pertaining to RWIV are scarce due to the complexity of the problem and the need to couple models for the thin film of water to an unsteady aerodynamic field and the structural dynamics of the cable. Previous work by two of the authors (Robertson and Taylor, 2007) using the aerodynamic solver DIVEX investigated the effect of both static and circumferentially oscillating 'artificial rivulets' on the unsteady aerodynamic field. This determined that for a static 'artificial' rivulet the system responds in a manner reminiscent of classical galloping with rivulet location being the dominant influence on the aerodynamic characteristics, which is consistent with previous experimental research of $\mathrm{Gu}$ and Huang (2008) and Matsumoto et al. (2007), while with an oscillating rivulet the response induced was significantly different and more complex. In future the authors plan to couple that solver to another which is capable of predicting the evolution of a thin-film of fluid given an aerodynamic loading, such that the temporal influence of rivulet evolution on the aerodynamic field and the influence of the aerodynamic field on rivulet formation and evolution can be determined. It is hoped that this new model will provide useful information to help in the development of a better understanding of the underlying RWIV mechanism, which is as yet unknown, and act as an initial step on the path to numerical simulation of RWIV, which is relatively undeveloped compared with experimental and analytical modelling. However, to accomplish this, a thin-film solver must first be constructed and validated.

Reisfeld and Bankoff (1992) derived an equation describing the evolution of a two-dimensional thin film of fluid on the outside of a horizontal cylinder subject to gravity, surface tension, thermo-capillary and long-range inter-molecular forces, and following this approach Peil and Dreyer (2007) and Lemaitre et al. (2007) derived a corresponding equation for a film on a cylinder subject to gravity, surface tension, wind shear and the motion of the cylinder. Solving the latter equation numerically, Peil and Dreyer (2007) computed the spreading of an initially parabolic rivulet, and Lemaitre et al. (2007) showed that a film of initially uniform thickness will develop 'bulges' ('rivulets') in the neighbourhood of the points where the air flow on a dry cylinder would separate; moreover, they concluded that shear and pressure forces are of similar importance in generating these bulges. A similar approach to that of Peil and Dreyer (2007) and Lemaitre et al. (2007) is adopted here in an analysis of the behaviour of a thin fluid film on a cylinder in an external flow, except that here the latter is determined separately, and is then coupled to the film flow via the normal stresses and shear stresses that it exerts.

The present paper describes a numerical model for the simulation of rivulet formation and evolution which has been developed by the Departments of Mechanical Engineering and Mathematics at the University of Strathclyde. This utilises the lubrication approximation to develop a two-dimensional model that simulates the interaction between a given static external aerodynamic field and a thin film of rain water on a horizontal circular cylinder. Using this method the governing evolution equation for the thickness of the film subject to normal and tangential stresses that result from this aerodynamic field in addition to the forces of surface tension and gravity is derived. The bespoke pseudo-spectral solver developed to solve this equation is then verified against previous analytical and numerical investigations. A selection of possible combinations of loading, namely pressure, shear, surface tension and gravity, are investigated, and the effects that these have on the film evolution are outlined. The temporal evolutions of the films are presented for the first time, and the effects of the magnitude and the distribution of the external aerodynamic loading are examined over a Reynolds number range representative for RWIV. 


\section{Model}

Two-dimensional, unsteady flow of a thin film of incompressible viscous fluid with uniform dynamic viscosity $\mu$ and density $\rho$ on the outer surface of a stationary horizontal circular cylinder of radius $R$ is considered. This restricts all loading to act purely within the two-dimensional system defined and is in line with the previous numerical and analytical studies into aspects of RWIV by Lemaitre et al. (2007), Yamaguchi (1990) and Gu and Huang (2008). Should an inclined cylinder be considered, then the effective component of gravity would be reduced; however, this would introduce a gravity-driven flow down the cylinder, and would also introduce uncertainties regarding the effective cylinder cross-section and the resulting aerodynamic loading. As Fig. 1 shows, the free surface of this film is subject to a prescribed pressure, $P=P(\theta, t)$, and a prescribed shear, $T=T(\theta, t)$, exerted by the external aerodynamic field, which are functions of clockwise angle from the windward (left-hand) horizontal, $\theta\left(0 \leq \theta \leq 360^{\circ}\right)$ and time $t$.

\subsection{Model description}

We take the film to be thin, its aspect ratio $\varepsilon$ (defined by $\varepsilon=H / R$, where $H$ denotes a typical film thickness) satisfying $\varepsilon \ll 1$.

We denote the fluid velocity and pressure by $\mathbf{u}$ and $p$, respectively. Initially we refer the description to polar coordinates $r, \theta, z$ with the $z$ axis along the axis of the cylinder; then the surface of the cylinder is given by $r=R$. We denote the film thickness by $h=h(\theta, t)$ (unknown a priori); then the free surface of the film is given by $r=R+h$. Near any station $\theta=$ constant we may alternatively refer the description to a local Cartesian coordinate system $O x y z$ with $O x$ tangential to the cylinder (increasing in the direction of increasing $\theta$, so that $x=R \theta+$ constant) and $O y$ along the outward normal to the cylinder, with $y$ defined by $y=r-R$, so that the cylinder is at $y=0$ and the free surface is at $y=h$. In the latter coordinate system the governing mass-conservation and Navier-Stokes equations give, at leading order in $\varepsilon$,

$$
\begin{aligned}
& u_{x}+v_{y}=0, \\
& 0=-p_{x}-\rho g \cos \theta+\mu u_{y y}, \\
& 0=-p_{y},
\end{aligned}
$$

where subscripts denote differentiation, and we have written

$$
\mathbf{u}=u \mathbf{i}+v \mathbf{j}, \quad \mathbf{g}=-g(\mathbf{i} \cos \theta+\mathbf{j} \sin \theta) .
$$

In Eqs. (2) and (3) the inertia terms have been neglected; this is valid provided that the film Reynolds number $\hat{R} e$ (defined by $\hat{R e}=\hat{U} R / v$, where $v=\mu / \rho$ and $\hat{U}$ are the kinematic viscosity and a typical velocity of the film, respectively) is such that $\varepsilon^{2} \hat{\mathrm{R}} \mathrm{e} \ll 1$. Also since the film is thin $(\varepsilon \ll 1)$, terms such as $u_{x x}$ and $v_{y y}$ in the momentum balances are negligible, as is a contribution $\rho g \sin \theta$ in (3). Eqs. (1)-(3) are subject to the no-slip and no-penetration conditions on the cylinder:

$$
u=v=0 \quad \text { on } y=0
$$

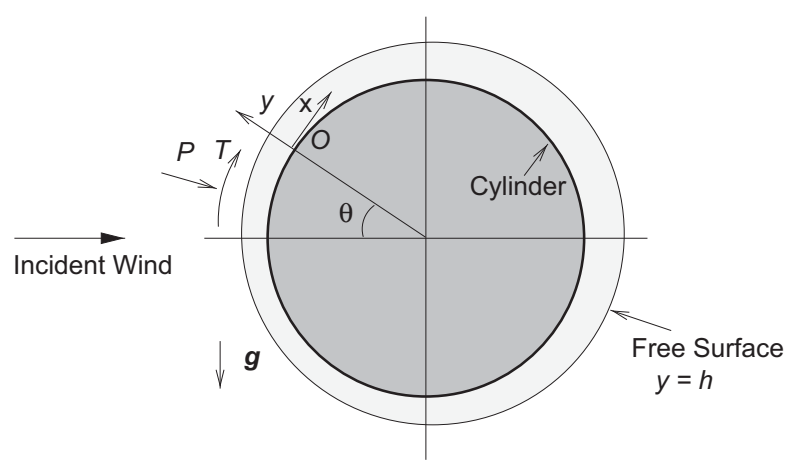

Fig. 1. A thin fluid film on a horizontal cylinder. 
and, at the free surface, to the kinematic condition

$$
v=h_{t}+u h_{x} \quad \text { on } y=h,
$$

the tangential stress condition

$$
\mu u_{y}=T \quad \text { on } y=h,
$$

and the normal stress condition

$$
p=\gamma \kappa+P \text { on } y=h,
$$

where $\gamma$ is the coefficient of surface tension and $\kappa$ is the mean curvature of the free surface, given to first order by

$$
\kappa=\frac{1}{R}-\frac{1}{R^{2}}\left(h+h_{\theta \theta}\right)
$$

The azimuthal volume flux of fluid in the film is given by

$$
Q=\int_{0}^{h} u \mathrm{~d} y,
$$

and using this and (1) we may replace (6) by the local conservation law

$$
h_{t}+Q_{x}=0 \text {. }
$$

\subsection{Evolution equation for $h(\theta, t)$}

Integrating (3) subject to (8) we obtain

$$
p=\gamma \kappa+P
$$

(independent of $y$ ), and then integrating (2) with respect to $y$ subject to (5) and (7) we obtain

$$
u=-\frac{1}{2 \mu}\left(\rho g \cos \theta+p_{x}\right)\left(2 h y-y^{2}\right)+\frac{T y}{\mu} .
$$

Therefore, from (10)

$$
Q=-\frac{1}{3 \mu}\left(\rho g \cos \theta+p_{x}\right) h^{3}+\frac{T h^{2}}{2 \mu} .
$$

Finally, substituting (14) into (11) and using (9) and (12) leads to the evolution equation for $h(\theta, t)$ :

$$
h_{t}+\left(\frac{T h^{2}}{2 \mu R}\right)_{\theta}-\left[\frac{h^{3}}{3 \mu R}\left(\rho g \cos \theta-\frac{\gamma}{R^{3}}\left(h+h_{\theta \theta}\right)_{\theta}+\frac{P_{\theta}}{R}\right)\right]_{\theta}=0 .
$$

This equation is to be solved subject to an initial condition of the form $h(\theta, 0)=h_{0}(\theta)$, where $h_{0}(\theta)$ is the initial thickness of the film. For definiteness in the present work we choose an initially uniform film $h_{0}=$ constant, and allow the film to evolve according to (15) to see if rivulets develop.

The present evolution equation (15) agrees with the corresponding equation given by Lemaitre et al. (2007) in the case of flow over a stationary cylinder, and with the earlier equation of Reisfeld and Bankoff (1992) for the case without aerodynamic loading. Given the nature of the problem, the same assumptions regarding the thin film and the boundary conditions were made here as were made by Lemaitre et al. (2007), and so the evolution equation (15) is essentially the same as that given by Lemaitre et al. (2007); however, unlike in the previous work, we present (15) in a dimensional rather than nondimensional format, this being done to facilitate the future coupling to an unsteady aerodynamic solver.

\section{Numerical solver}

As the evolution equation (15) is a fourth order, non-linear, non-constant coefficient partial differential equation, it cannot, in general, be solved analytically. Therefore, a pseudo-spectral (or collocation) method solver using an $\mathrm{N}$-point Fourier spectral mode in space and a fourth order Adams-Bashforth time-marching algorithm was constructed. This numerical method was chosen specifically because of the periodic, continuous nature of the problem over the interval 
$\left[0^{\circ}, 360^{\circ}\right)$ and the rapid rate of convergence it provides to the solution, given the presumed smoothness of the final result.

As with the mathematical formulation there are again distinct similarities between the present numerical solver and those of Reisfeld and Bankoff (1992) and Lemaitre et al. (2007), reflecting the fact that the Fourier pseudo-spectral method is so well suited to the problem under examination. That said, the present solver was created independently for use within a wider context, particularly the future coupling previously mentioned. In addition to examining a wider range of cases than previously studied, including how the Reynolds number and the magnitude and form of the aerodynamic loading affect rivulet formation and evolution, the present study also reports this information in a temporal manner for the first time. Moreover, at a lower level it should act as confirmation of the applicability of the method itself, and provide independent validation of the results of Reisfeld and Bankoff (1992) and Lemaitre et al. (2007).

\subsection{Parameter selection}

Efficient usage of the fast Fourier transform (FFT) requires $N$ to be a power of 2 (Fornberg, 1996). Through a computational convergence study of a problem that will be discussed in Section 4.1, an equi-spaced distribution of $N=128$ points was found to provide the optimum compromise between run-time and stability, and spatial resolution for the present code. A partial summary of this convergence study is given in Table 1. This shows that the CPU time required to solve the problem for a given number of timesteps, in this instance $1 \times 10^{5}$, is approximately proportional to the number of points used in the calculation $(t \propto N)$, while for a given timestep size, $\Delta t$, the solution was also found to resolve to a greater final time for fewer points, although a limit was reached and no simple correlation was found. These final times at which a resolved solution could be obtained, $\tau_{\max }$, where a higher value indicates a more stable solution, are also given in Table 1 for a timestep of $\Delta t=1.0 \times 10^{-6} \mathrm{~s}$. These use a reduced time $\tau=g h_{0}^{2} t / 3 v R$, as defined by Reisfeld and Bankoff (1992); the solution becomes singular as $\tau \rightarrow 0.5$, there being an unbounded growth in film height at the lowest point of the cylinder, $h\left(270^{\circ}, \tau\right)$. Although a faster, more stable solution can be found with fewer points, this reduces overall spatial resolution. Therefore, given the values in Table 1 and the overall resolution required, a value of $N=128$ was chosen.

A similar convergence study to that presented in Table 1 was undertaken with regard to timestep size for a given number of points. Although the details are omitted, this revealed a time-step $\Delta t=5.0 \times 10^{-7} \mathrm{~s}$ to be sufficient for $N=128$. From these studies, the number of points and time-step were fixed at $N=128$ and $\Delta t=5.0 \times 10^{-7} \mathrm{~s}$, respectively, throughout this investigation.

Standard values for gravity and the properties at an air-water interface at $20^{\circ} \mathrm{C}$ were selected for use throughout the present study and are listed in Table 2, while other parameter values, such as incident wind velocity, $V$, were chosen to represent typical values for RWIV and to ensure that the ratio of initial film thickness to cylinder radius $h_{0} / R=6.3 \times 10^{-3}$ was consistent with those of previous experimental and computational studies by Flamand (1995) and Lemaitre et al. (2007). With these parameter values, the Reynolds number implemented was a subcritical value typical for $\mathrm{RWIV}$ of $\mathrm{Re} \simeq 1 \times 10^{5}$, where $\mathrm{Re}=V D / v^{*}$ concerns the external fluid, in this instance air (rather than the thin film), and is defined in terms of a typical incident wind speed $V$, the cylinder diameter $D$ and the kinematic viscosity, $v^{*}$.

The distributions of pressure $P$ and shear $T$ due to the external aerodynamic field were assumed to be constant in time and thus functions only of angle, i.e. $P=P(\theta)$ and $T=T(\theta)$. These are based upon the time averaged pressure and friction coefficients, $C_{P}$ and $C_{F}$, for the external aerodynamic field around a dry cylinder, values for which were determined experimentally by Achenbach (1968) at a Reynolds number similar to that under investigation here of $\mathrm{Re}=1 \times 10^{5}$. Although, as previously outlined, future developments of the present work will use a coupled aerodynamic solver to ascertain the aerodynamic coefficients $C_{P}$ and $C_{F}$ based upon the present evolutionary shape, this is not the focus of the present study. Thus, although the assumption that the formation of rivulets will not affect the aerodynamic profile is limited and strictly valid only for a uniform film, it is seen as applicable for the present study.

Table 1

Summary of the computational convergence study.

\begin{tabular}{|c|c|c|c|c|c|}
\hline Number of points, $N$ & 32 & 64 & 128 & 256 & 512 \\
\hline CPU time (s) & 3.108 & 4.632 & 9.445 & 18.137 & 36.222 \\
\hline Final resolved time $\tau_{\max }$ & 0.487 & 0.485 & 0.479 & 0.448 & 0.227 \\
\hline
\end{tabular}


Table 2

Values of the standard parameters used in the numerical calculations.

\begin{tabular}{lc}
\hline Parameter & Value \\
\hline Cylinder radius, $R$ & $0.08 \mathrm{~m}$ \\
Initial film height, $h_{0}$ & $5 \times 10^{-4} \mathrm{~m}$ \\
Gravity, $g$ & $9.806 \mathrm{~m} / \mathrm{s}^{2}$ \\
Density of water, $\rho$ & $1000 \mathrm{~kg} / \mathrm{m}^{3}$ \\
Dynamic viscosity of water, $\mu$ & $1.002 \times 10^{-3} \mathrm{~N} \mathrm{~s} / \mathrm{m}^{2}$ \\
Surface tension of water, $\gamma$ & $72 \times 10^{-3} \mathrm{~N} / \mathrm{m}$ \\
Density of air, $\rho^{*}$ & $1.19 \mathrm{~kg} / \mathrm{m}^{3}$ \\
Dynamic viscosity of air, $\mu^{*}$ & $1.82 \times 10^{-5} \mathrm{~N} \mathrm{~s} / \mathrm{m}$ \\
Incident wind speed, $V$ & $11.0 \mathrm{~m} / \mathrm{s}$ \\
\hline
\end{tabular}

One of the limitations of the pseudo-spectral method is its susceptibility to aliasing of high frequencies, especially in the non-linear terms present in the evolution equation (15). To avoid this problem, and to maintain a high convergence rate (for the pseudo-spectral method this is greater for analytic functions), a truncated Fourier series representation of the aerodynamic coefficients $C_{P}$ and $C_{F}$ of the form

$$
C_{P}, C_{F}=\frac{a_{0}}{2}+\sum_{k=1}^{n}\left[a_{k} \cos (k \theta)+b_{k} \sin (k \theta)\right]
$$

was used in the analysis. To ensure that the number of terms chosen in (16) is sufficient to reproduce the variation of the aerodynamic coefficients around the cylinder accurately, a brief study of the number of terms required, $n$, was undertaken. The results of this investigation for both coefficients were very similar and hence only a summary of the error due to series truncation for the pressure coefficient $C_{P}$ is given in Fig. 2. This revealed that the coefficient of determination, $R^{2}$ (not to be confused with the cylinder radius $R$ ) was high, $R^{2}>0.985$, throughout the range of number of terms investigated, $[2,30]$. It also showed that the percentage error between the truncated series and experimentally determined values at a specific value of $\theta$ for the majority of the range investigated was small. This, however, was not true at either of the two sharp peaks in the $C_{P}$ profile at $\theta \simeq 70^{\circ}$ and $\theta \simeq 290^{\circ}$, where the largest errors occurred (Figs. 2 and 3). At these points the percentage error began to plateau at a minimum value only after $n \geq 20$ terms, and therefore the first twenty terms were chosen to represent the two coefficients. A comparison of the final truncated Fourier series (16) using $n=20$ with the original experimental coefficients of Achenbach (1968) is shown in Fig. 3. Note that the friction coefficient shown, $\bar{C}_{F}=C_{F} / \max \left(C_{F}\right)$, is a normalised version of $C_{F}$. For the numerical investigation, these series representations of $C_{P}$ and $C_{F}$ were then appropriately scaled such that the maximum values of pressure and shear loadings matched those used by Lemaitre et al. (2007), thus allowing direct comparisons between the results.

\section{Model verification}

Verification of the numerical procedure was undertaken by comparison of the present results with analytical solutions under two specific loading cases, namely:

(i) gravity and surface-tension loading $(T \equiv 0$ and $P \equiv 0)$ and

(ii) only constant shear loading $(T(\theta) \equiv$ constant, with $P \equiv 0, \gamma=0$ and $g=0)$.

\subsection{Gravity and surface-tension loading}

Initial verification was undertaken by comparison of the present results with the work of Reisfeld and Bankoff (1992) who investigated the heating or cooling of a thin viscous film of fluid on the outer surface of a circular cylinder, subject to the forces of gravity and surface tension. Under isothermal conditions this work corresponds directly to the present study when no aerodynamic loading is considered (i.e. when pressure $P \equiv 0$ and shear $T \equiv 0$ ). In this case, the evolution equation (15) thus represents a direct competition between the effects of gravity, viscosity and surface tension on the 


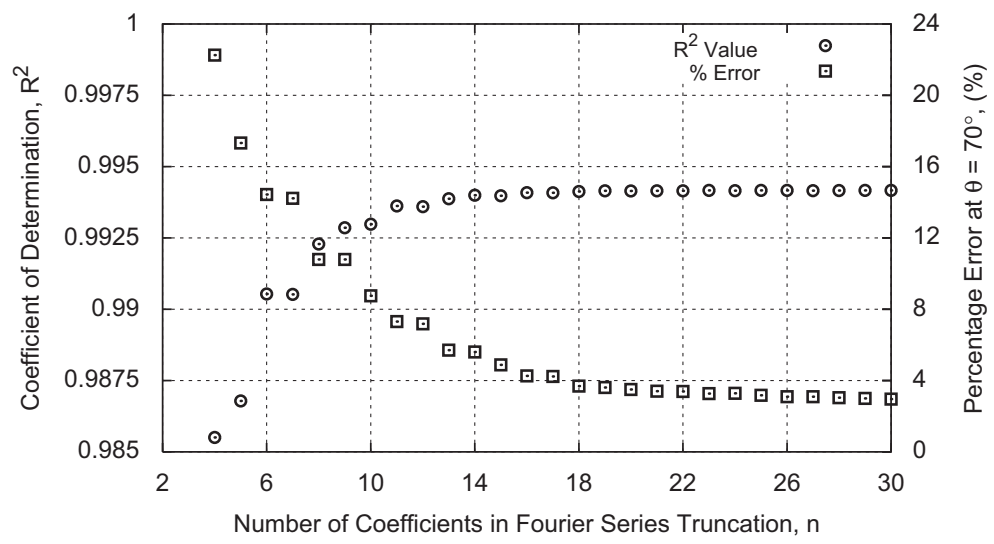

Fig. 2. Variation of the coefficient of determination $R^{2}$ and percentage error at $\theta=70^{\circ}$ with the number of terms, $n$, in the truncated Fourier series representation of the pressure coefficient $C_{P}$.

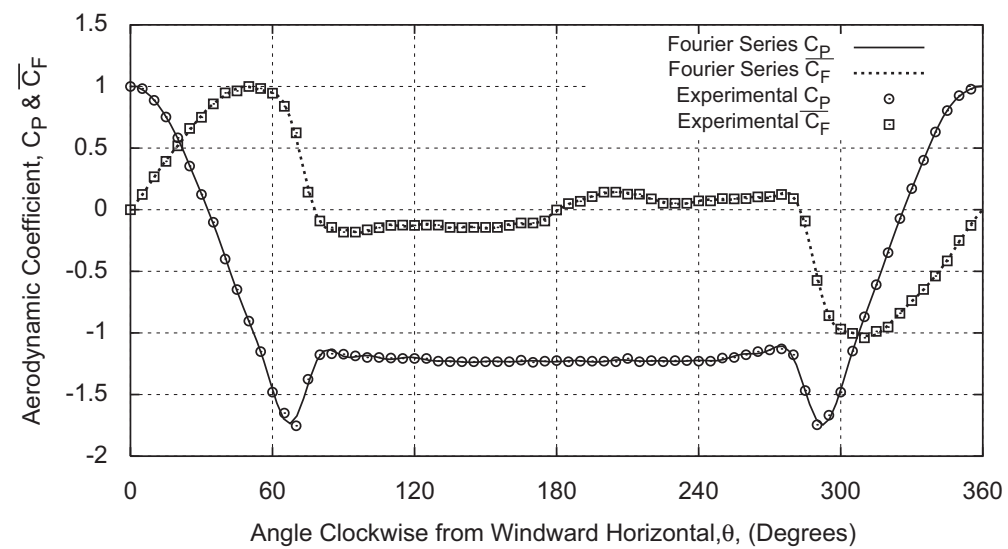

Fig. 3. Aerodynamic coefficients $C_{P}$ and $C_{F}$ used in the numerical calculations and those measured experimentally by Achenbach (1968) at $\operatorname{Re}=1 \times 10^{5}$.

film. In particular, in the limit of a very large Bond number Bo $=\rho g R^{3} / h_{0} \gamma \rightarrow \infty$, Reisfeld and Bankoff (1992) determined an analytical solution for the film height around the cylinder in terms of a reduced time $\tau=g h_{0}^{2} t / 3 v R$, subject to the initial condition $h(\theta, 0)=1$, namely (in the present notation)

$$
h(\theta, \tau)= \begin{cases}(1+2 \tau)^{-1 / 2} & \text { for } \theta=90^{\circ}, \\ (1-2 \tau)^{-1 / 2} & \text { for } \theta=270^{\circ}, \\ \left(\cos \theta_{0} / \cos \theta\right)^{1 / 3} & \text { for } 0^{\circ} \leq \theta<360^{\circ}, \theta \neq 90^{\circ}, 270^{\circ},\end{cases}
$$

where $\theta_{0}=\theta_{0}(\theta, \tau)$ is determined from

$$
F\left(g(\theta), \sin 75^{\circ}\right)-F\left(g\left(\theta_{0}\right), \sin 75^{\circ}\right)+2(3)^{1 / 4}\left(\cos \theta_{0}\right)^{2 / 3} \tau=0,
$$

in which $F(\phi, k)$ is an incomplete elliptic integral of the first kind defined by

$$
F(\phi, k)=\int_{0}^{\phi} \frac{d x}{\sqrt{1-k^{2} \sin ^{2} x}},
$$

and $g(\psi)$ satisfies

$$
\cos g(\psi)=\frac{\sqrt{3}-1+(\cos \psi)^{2 / 3}}{\sqrt{3}+1-(\cos \psi)^{2 / 3}} .
$$




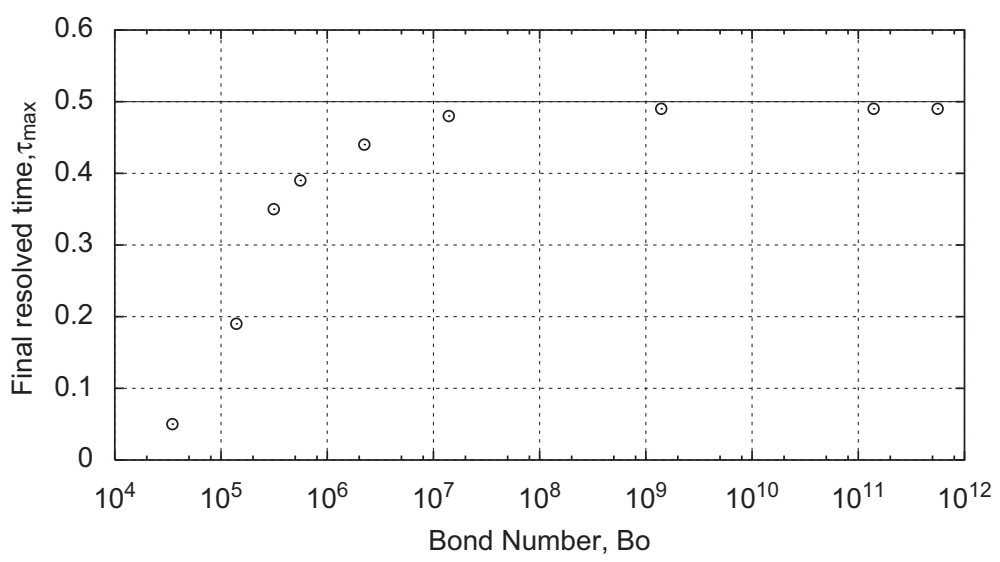

Fig. 4. Final resolved time, $\tau_{\max }$ as a function of Bo.

To effect a valid comparison between the present work and the analytical solution a study was undertaken to determine practical values for the numerical representation of Bo $\rightarrow \infty$. To achieve this, the values of cylinder radius $R$ and initial film thickness $h_{0}$ were varied whilst maintaining a constant ratio of $h_{0} / R=6.3 \times 10^{-3}$. All other parameters were kept at the original values as listed in Table 2. The results of this study are shown in Fig. 4 which plots the final resolved time, $\tau_{\max }$, as a function of Bo. Eq. (17) predicts that the film height at the lowest point, $h\left(270^{\circ}, \tau\right)$, becomes singular as $\tau \rightarrow 0.5$; therefore $\tau_{\max } \rightarrow 0.5^{-}$as Bo $\rightarrow \infty$, and as illustrated in Fig. 4, in practice Bo $\geq 1 \times 10^{7}$ is sufficient to represent this.

Using the parameters chosen for RWIV presented in Table 2 results in Bo $=1.4 \times 10^{5}$, which is below the range for the exact solution (17) to be applicable. Therefore, for the purposes of verification of the numerical model, the initial height of film, $h_{0}$, and radius of the cylinder, $R$, were each increased by an order of magnitude, to 0.8 and $0.005 \mathrm{~m}$, which gives a corresponding Bond number of $1.4 \times 10^{7}$. To verify the pseudo-spectral solver for Bo $=1.4 \times 10^{7}$, the computational results were compared with the analytical solution of Reisfeld and Bankoff (1992) given in (17)-(20). Fig. 5 shows the evolution of the normalised film thickness, $h / h_{0}$, at the highest and lowest points of the cylinder, $\theta=90^{\circ}$ and $270^{\circ}$, with reduced time $\tau$. The results show excellent agreement up to $\tau \simeq 0.45$ where the numerical calculations become increasingly less accurate and more unstable due to the singularity that arises at $\theta=270^{\circ}$ as $\tau \rightarrow 0.5$.

To verify the procedure over the entire cylinder surface $\left[0^{\circ}, 360^{\circ}\right)$ and not just at two specific points $\left(\theta=90^{\circ}\right.$ and $\left.270^{\circ}\right)$, the present results were also compared to the analytical solution (17) for all $\theta$ at specific instants of reduced time. Fig. 6 shows one such comparison, specifically $\tau=0.4$, which is typical of the excellent agreement for all values of $\tau \leq 0.45$.

\subsection{Constant shear loading only}

In contrast to the previous section, the present subsection examines the response to loading that arises from the aerodynamic field, namely that of a purely shear-driven flow subject to a constant surface shear $T$. Details of the solution of this problem, along with a note on the limits of its applicability, are presented in the appendix, in which it is shown that the (implicit) solution for $h(\theta, t)$ satisfying the initial condition $h(\theta, 0)=h_{0}(\theta)$ is given by

$$
h=h_{0}\left(\theta-\frac{T h}{\mu R} t\right) \text {. }
$$

If we take the initial profile to be uniform, $h_{0}(\theta)=$ constant, under the action of a constant shear the film retains this uniform profile for all time, which is rather uninteresting. Therefore, we will examine a simple non-uniform initial profile, namely, $h_{0}(\theta)=H(1-a \cos \theta)$ with $H>0$ and $|a|<1$. Then, (21) gives the implicit equation

$$
\frac{h}{H}=1-a \cos \left(\theta-\frac{T h}{\mu R} t\right),
$$

which determines $h$ as a function of $\theta$ and $t$. Using the parameters from Table 2 and an initial film thickness profile with $H=0.0005 \mathrm{~m}$ and $a=0.1$ then, as shown in the appendix, the analytical solution (21) predicts that the profile of the free surface will 'break' at time $t=1.6032 \mathrm{~s}$ and position $\theta=\theta_{s} \simeq 123^{\circ}$. 


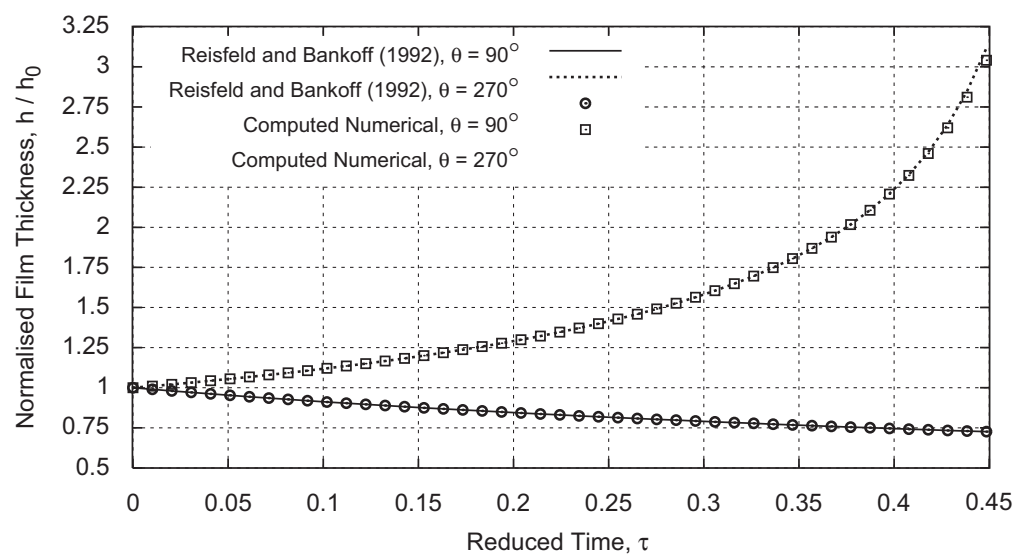

Fig. 5. Comparison of the analytical solution of Reisfeld and Bankoff (1992) given in Eqs. (17)-(20) with computed numerical results for normalised film height on cylinder at $\mathrm{Bo}=1.4 \times 10^{7}$ : temporal evolution at top $\left(\theta=90^{\circ}\right)$ and bottom $\left(\theta=270^{\circ}\right)$ of the cylinder.

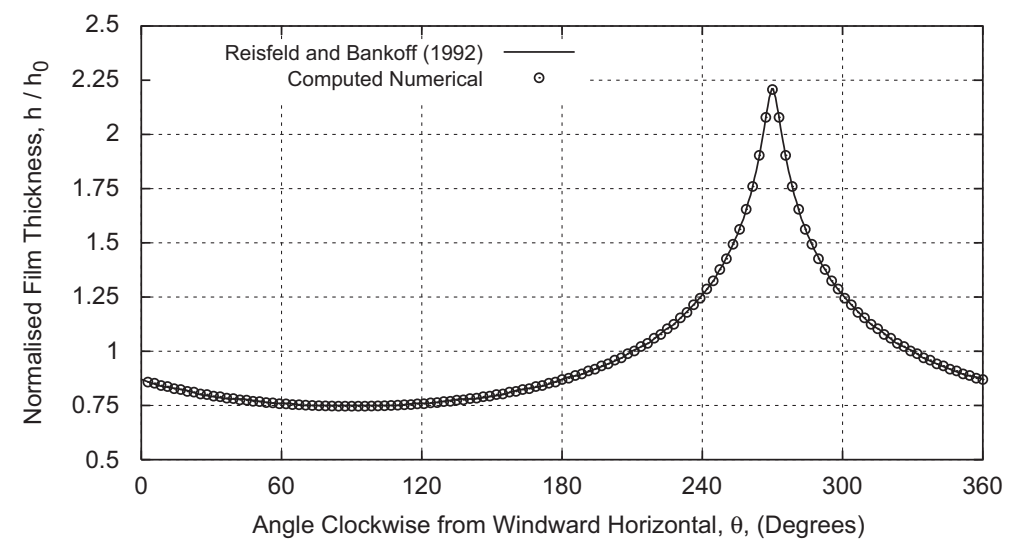

Fig. 6. Comparison of the analytical solution of Reisfeld and Bankoff (1992) given in Eqs. (17)-(20) with computed numerical results for normalised film height on cylinder at $\mathrm{Bo}=1.4 \times 10^{7}$ : spatial distribution $\left(0 \leq \theta \leq 360^{\circ}\right)$ at $\tau=0.4$.

This was indeed found to be the case, as can be seen in Fig. 7, which shows a comparison between the present results and the theoretical profile (21) at this 'breaking' time. Indeed this excellent agreement was found for all times $t<1.6032$. Fig. 7 also displays the comparison between the analytical and the presently determined profiles at an earlier instant, $t=1 \mathrm{~s}$, typical for all times up to this 'breaking' time.

As was true for the previous verification study in Section 4.1, the excellent agreement between the present results and the analytical solution was found both for specific instants in time and for the temporal evolution at specific points on the surface. The latter can be seen in Fig. 8 which shows the temporal evolution of normalised film height at two specific points on the top and bottom of the cylinder, namely $\theta=90^{\circ}$ and $\theta=270^{\circ}$, which are representative for the entire cylinder.

\section{Results}

Four different combinations of pressure $P$, shear $T$, surface tension $\gamma$ and gravity $g$ loading were considered in this study. These were

(1) gravity and surface tension $(T \equiv 0$ and $P \equiv 0)$,

(2) shear and surface tension $(P \equiv 0$ and $g=0)$, 


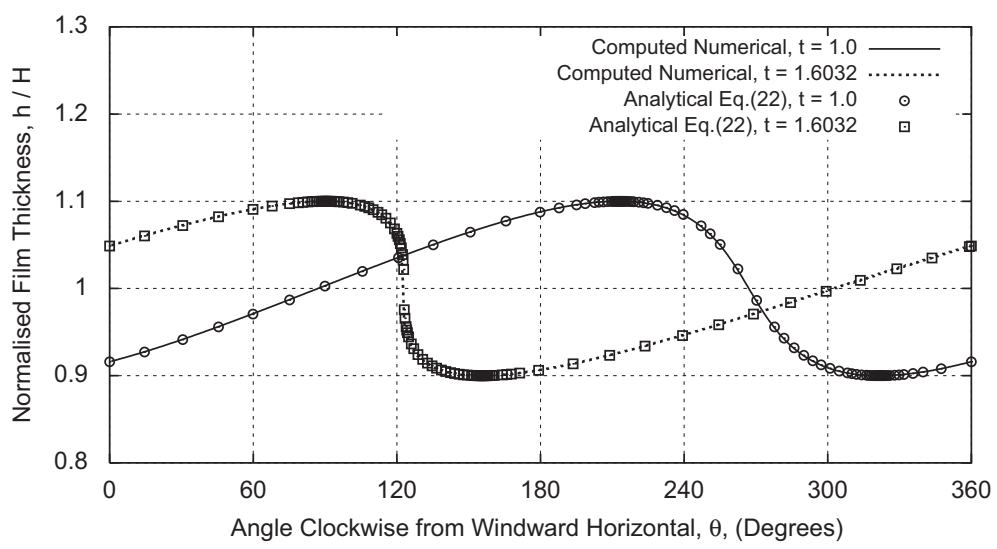

Fig. 7. Comparison of analytical solution for constant shear, Eq. (22), with computed numerical results for spatial distribution of normalised film height on cylinder at $t=1$ and $1.6032 \mathrm{~s}$.

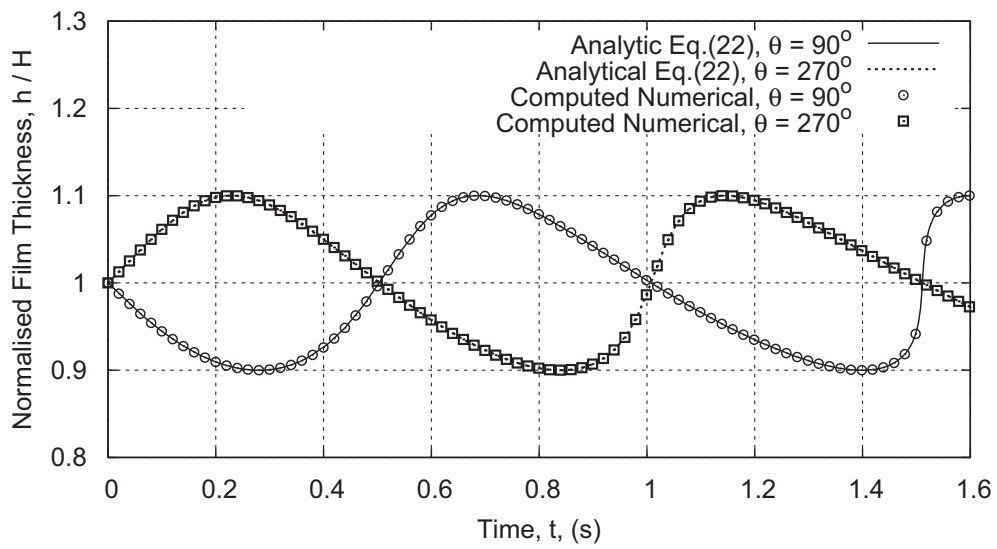

Fig. 8. Comparison of analytical solution for constant shear, Eq. (22), with computed numerical results for normalised film height for temporal evolution at top $\left(\theta=90^{\circ}\right)$ and bottom $\left(\theta=270^{\circ}\right)$ of the cylinder.

(3) pressure and surface tension $(T \equiv 0$ and $g=0)$, and

(4) full loading $(P, T, g$ and $\gamma \neq 0)$,

where the pressure and shear loads represent the normal and tangential components of the surface stress which result from the aerodynamic field. Several sub-cases such as how the magnitude and form of aerodynamic loading, and the Reynolds number affect the evolutionary response are examined for the first time. The results are also presented in a temporal manner for the first time.

\subsection{Gravity and surface-tension loading}

The combination of gravity and surface-tension loading was examined for the realistic values of $h_{0}$ and $R$ shown in Table 2. Fig. 9 illustrates the temporal evolution of film thickness $h$ as a function of $\theta$ and provides a clear representation of the film 'thinning' on the upper surface of the cylinder and a 'spike' in the film thickness growing on the lower surface, and the rates at which these occur. The fluid that accumulates at the base of the cylinder increases in depth until the lubrication approximation is violated and the theoretical method is no longer valid, which is consistent with the analytical solution (17), the computational work of Reisfeld and Bankoff (1992) and the verification study in the previous section.

A study into how the variation of the relative magnitude of the loadings due to gravity or surface tension affect the evolutionary response was undertaken by varying the magnitude of the gravity number, $G=g h_{0}^{3} / 3 v^{2}$, which measures 
the ratio of gravitational to viscous forces. The results showed that, provided Bo is considerably larger than $1 \times 10^{5}$, altering the relative effects of loading due to gravity or surface tension through variation of $G$ by two orders of magnitude does not significantly change the solution at the same value of reduced time, $\tau$; rather this alters only the real time $t$ taken to arrive at that solution. This is due to the dominance of gravity over surface-tension effects at these

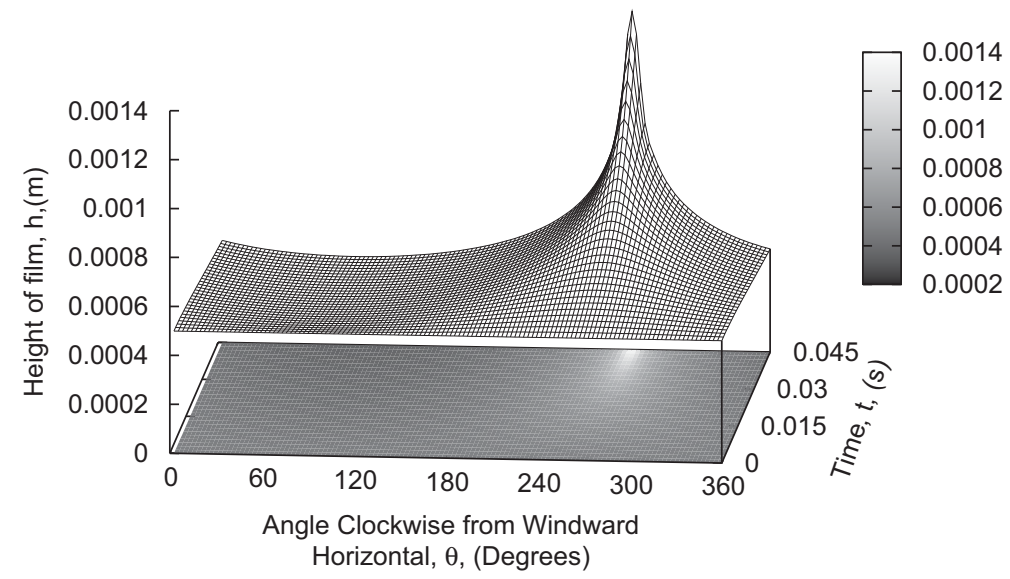

Fig. 9. Numerical prediction of temporal evolution of the film height in real time, under gravity and surface-tension effects only.

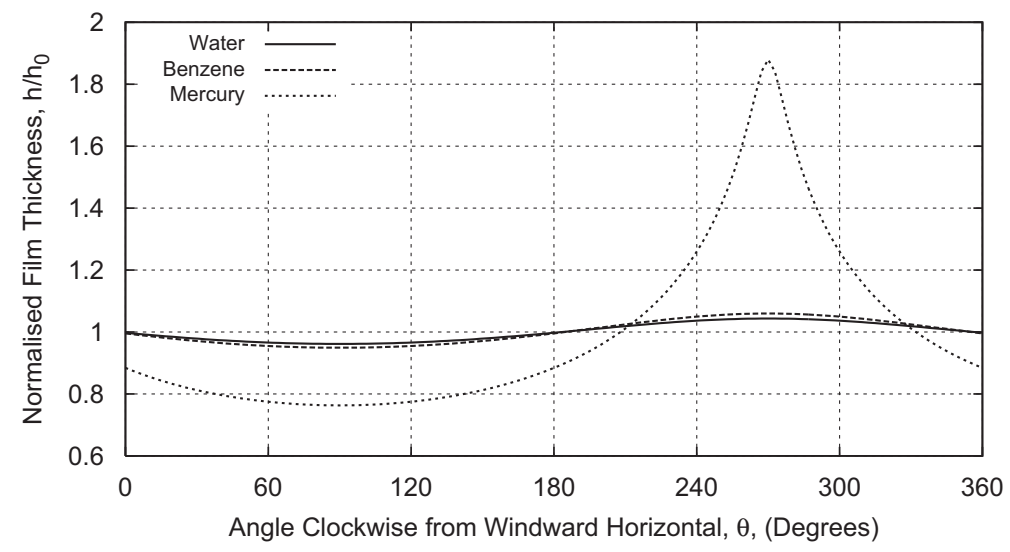

Fig. 10. Comparison of normalised film heights of water, benzene and mercury as functions of angle $\theta$ at real time $t=0.4 \times 10^{-3} \mathrm{~s}$.

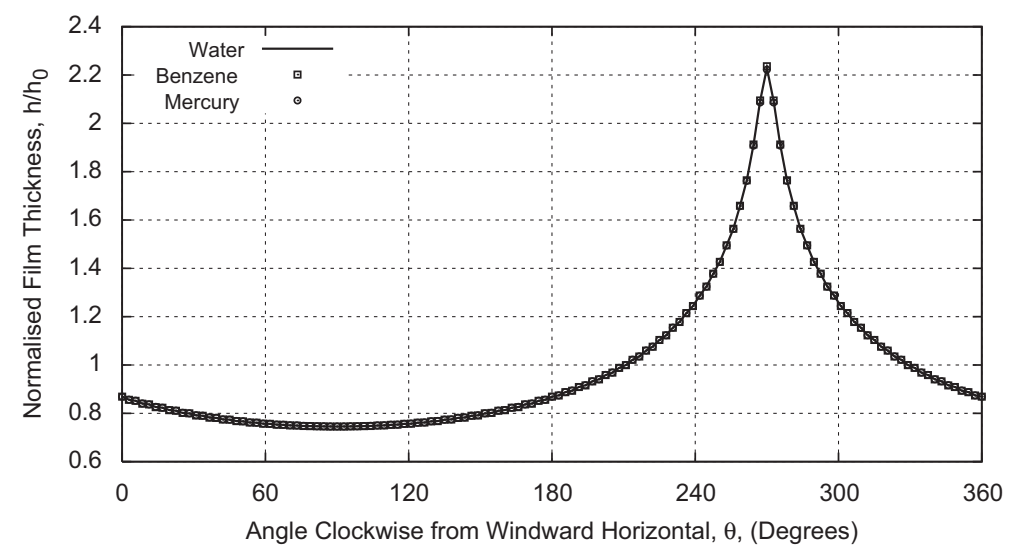

Fig. 11. Comparison of normalised film heights of water, benzene and mercury as functions of angle $\theta$ at reduced time $\tau=0.4$. 
gravity and Bond numbers. If Bo is considerably smaller than $1 \times 10^{5}$, this dominance is lost and the results change dramatically; this, however, is outwith the scope of the present work, and the reader is referred to Reisfeld and Bankoff (1992) who provide a fuller discussion of flows at lower Bo. To demonstrate this relation to the timescale used in the examination, Figs. 10 and 11 show the normalised height $h / h_{0}$ of three different fluids, water, benzene and mercury, at the same instant of real time $t$ and reduced time $\tau$, respectively. For each fluid the Bond number was approximately the same, but crucially the gravity number was distinctly different. The values of these parameters and the standard fluid properties at $20^{\circ} \mathrm{C}$ for each of these three fluids are given in Table 3. As in the initial verification study in Section 4.1 the values of cylinder radius $R$ and initial film thickness $h_{0}$ were again both increased by a factor of 10 to 0.8 and $0.005 \mathrm{~m}$, to ensure $\mathrm{Bo} \gg 1 \times 10^{5}$, specifically $\mathrm{Bo}=1.4 \times 10^{7}$.

Closer inspection of Fig. 10 reveals that, as mercury has a significantly greater gravity number $G=\mathcal{O}\left(10^{7}\right)$, this film evolves considerably quicker than those of the other two fluids, whereas because the gravity numbers of benzene and water are more closely matched and lower than that of mercury, namely $G=\mathcal{O}\left(10^{5}\right)$, these films develop at approximately the same rate, with water, which has the lowest value of $G$, being the slowest to evolve. Finally in the limiting case of gravity and surface tension being varied in such a way that Bo remains constant, the minimal variations at fixed reduced time $\tau$, shown in Fig. 11, are eliminated altogether.

\subsection{Shear and surface-tension loading}

The temporal evolution of the thin film under the effect of shear $T$ and surface tension $\gamma$ loading is shown in Fig. 12. Under this combination of loadings, two distinct symmetrically placed rivulets form, one each on the upper and lower surfaces. Fig. 12 shows the evolution at early times of these rivulets, whose location is just windward of the clockwise and anti-clockwise separation points for a dry cylinder at the same Re, which is consistent with the previous experimental analysis of Bosdogianni and Olivari (1996) and the numerical results of Lemaitre et al. (2007). Closer examination of the temporal evolution of the rivulets in Fig. 12 reveals that they are of approximately equal height, profile and growth rate; the minor discrepancies between the two can be attributed to a small asymmetry in the applied

Table 3

Parameters and fluid properties of water, benzene and mercury at $20^{\circ} \mathrm{C}$.

\begin{tabular}{llll}
\hline Property & Water & Benzene & Mercury \\
\hline Density, $\rho\left(\mathrm{kg} / \mathrm{m}^{3}\right)$ & 1000 & 880 & 13600 \\
Dynamic viscosity, $\mu\left(\mathrm{N} \mathrm{s} / \mathrm{m}^{2}\right)$ & $1.002 \times 10^{-3}$ & $0.656 \times 10^{-3}$ & $1.55 \times 10^{-3}$ \\
Surface tension, $\gamma(\mathrm{N} / \mathrm{m})$ & 0.072 & 0.039 & 0.51 \\
Bond number Bo & $1.4 \times 10^{7}$ & $3.1 \times 10^{7}$ & $2.7 \times 10^{7}$ \\
Gravity number $G$ & $4.1 \times 10^{5}$ & $7.4 \times 10^{5}$ & $3.2 \times 10^{7}$ \\
\hline
\end{tabular}

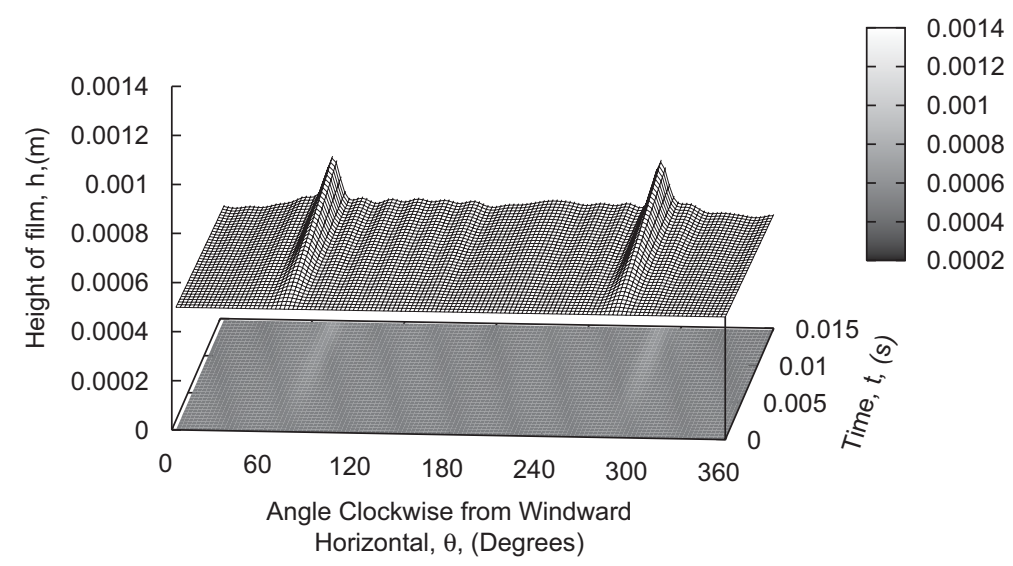

Fig. 12. Numerical prediction of the temporal evolution of film height in real time, under shear and surface-tension effects only. 
shear loading distribution which results from the faithful approximation of the aerodynamic coefficients determined experimentally by Achenbach (1968), since with a perfectly symmetric distribution of aerodynamic coefficients the two rivulets are indeed identical and symmetric about the incident wind.

The present results compare favourably with the only other computational results available for the same problem, namely those of Lemaitre et al. (2007), as illustrated in Fig. 13, which displays both sets of results at a specific instant in time, $t=6.9 \times 10^{-3} \mathrm{~s}$, the only time at which the results of Lemaitre are available. The minor differences between the two solutions can again be attributed to a variation in the distribution used for $C_{F}$ in the two schemes. To confirm this, the governing equation from Lemaitre et al. (2007) was used in the present solver with the present distribution of $C_{F}$. When these results are compared with those obtained using the present evolution equation (15), they were found to be identical over the entire time range under investigation. Fig. 14 illustrates this at a 'late' time of $t=34.0 \times 10^{-3} \mathrm{~s}$, by which point any differences between the results would be apparent. This confirms that differences between the present results and those of Lemaitre et al. (2007) are due to differences in the $C_{F}$ distribution. It also confirms that the evolution equations are equivalent, and that the independently developed numerical procedures to solve the evolution equations are also operating in a consistent manner. Whilst the comparison with previous numerical results is not a full validation, the level of agreement between independently derived methodologies verifies the approach that has been used.

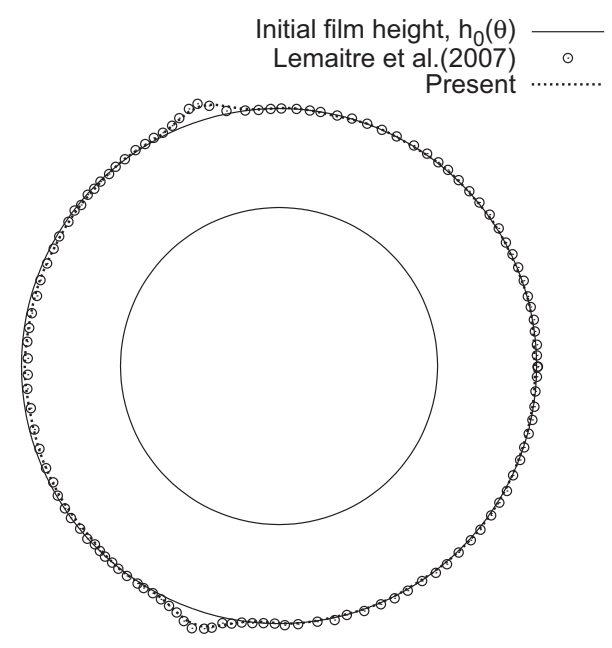

Fig. 13. Comparison of present numerical results with the results of Lemaitre et al. (2007) for variation of film height $(100 \times$ actual) at $t=6.9 \times 10^{-3} \mathrm{~s}$, under the effects of shear and surface tension, where the incident wind acts from the left.

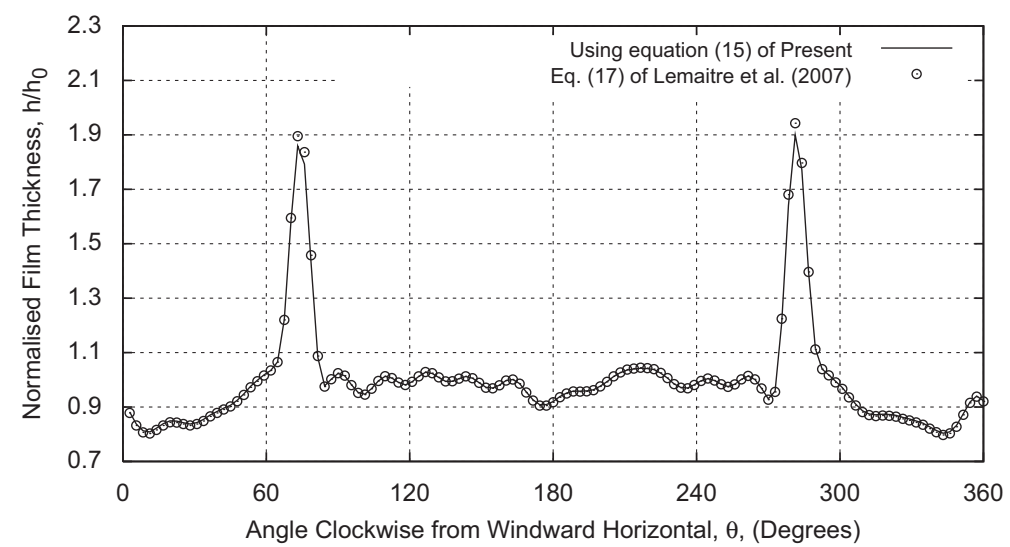

Fig. 14. Comparison of normalised film height under the effects of shear and surface tension from present numerical solver for evolution equation (15) and Eq. (17) of Lemaitre et al. (2007), using identical $C_{F}$ distribution, at $t=34.0 \times 10^{-3} \mathrm{~s}$. 


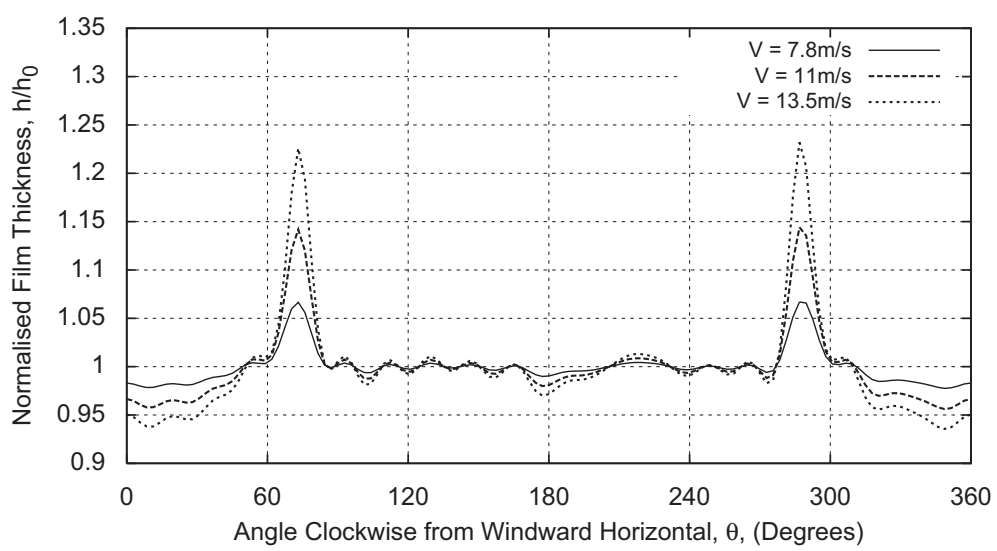

Fig. 15. Effect of varying shear-loading value $T$ through variation of the incident wind speed $V$ with constant distribution of $C_{F}$ on variation of normalised film height with angle from windward horizontal at $t=6.9 \times 10^{-3} \mathrm{~s}$.

\subsubsection{Effect of shear-stress magnitude}

To investigate the effect of the magnitude of the shear stress, a study was undertaken in which the distribution of $C_{F}$ was kept constant but the applied shear stress was altered through variation of the incident wind speed $V$. Several such values were investigated, although only three are reported here as these are representative of all the cases examined. These are the wind speed that has been used in the previous cases, $V=11 \mathrm{~m} / \mathrm{s}$, and two others $V=7.8$ and $13.5 \mathrm{~m} / \mathrm{s}$, which were specifically chosen as lower and upper limits to ensure that the applied shear stress was 0.5 and 1.5 times that of the original $(11 \mathrm{~m} / \mathrm{s})$ case and to ensure that these speeds were well within the typical range identified for RWIV $(\simeq 5-15 \mathrm{~m} / \mathrm{s})$.

The results showed that when only $V$ is altered the resulting film thickness evolution profile changes only in scale not in distribution; this is due to the dominance of the shear stress effects in comparison with those of surface tension for the specific parameters presently under investigation. Should different parameter values be used, namely values such that the magnitudes of surface-tension and shear loading are closer, it is expected that such a 'scaling' effect would no longer hold; that, however, is not the subject of the present study. Fig. 15 illustrates both this 'scaling' effect and the consistency of the angles $\theta$ at which the rivulets form on the upper and lower surfaces of the cylinder with varying incident wind speed at a time of $t=6.9 \times 10^{-3} \mathrm{~s}$. It should be noted that while the assumption that the distribution of $C_{F}$ does not change with $V$ (and hence Re) may be an idealisation, over the subcritical range of Reynolds number corresponding to the velocities studied, $0.82 \times 10^{5} \leq \mathrm{Re} \leq 1.43 \times 10^{5}$, any variation in the distribution of $C_{F}$ is expected to be slight, a point that is discussed in greater depth in Section 5.3.2.

\subsection{Pressure and surface-tension loading}

Under only pressure $P$ and surface tension $\gamma$ loading, the results show distinct similarities to those for the previous case of shear and surface-tension loading (Section 5.2.1). Here again approximately symmetric rivulets form just windward of the separation points on the top and bottom of a dry cylinder (see Figs. 16 and 17). These asymmetries in the rivulets again result from the reproduction of asymmetries in the applied $C_{P}$ profile, as was true for the previous shear case, with the temporal distribution of film height again becoming symmetric should a symmetric aerodynamic profile be used. In this instance, however, the locations of these rivulets are marginally windward of those formed in the shear and surface-tension case (Fig. 17), closer examination of which shows that the 'size' of the rivulets under the present pressure loading are approximately the same order of magnitude as those with the previous shear-loading condition. Given this and the similar rates of rivulet growth, which can be ascertained from Figs. 12 and 16, respectively, the indication is that the effects of pressure and shear loadings are of a similar magnitude under the present conditions, and that they are therefore of broadly equal importance. Here the definition of rivulet 'size' has been used loosely, as the differences in height and width of the rivulets formed between the shear and pressure loading cases is noticeable. However, the amount of additional fluid accumulated within each (based on the relative cross sectional areas) is approximately the same, namely $\simeq 1.25 \times 10^{-3}$ and $\simeq 1.72 \times 10^{-3} \mathrm{~mm}^{2}$ for the shear and pressure cases, respectively. The main reason for this variation in shape can be 


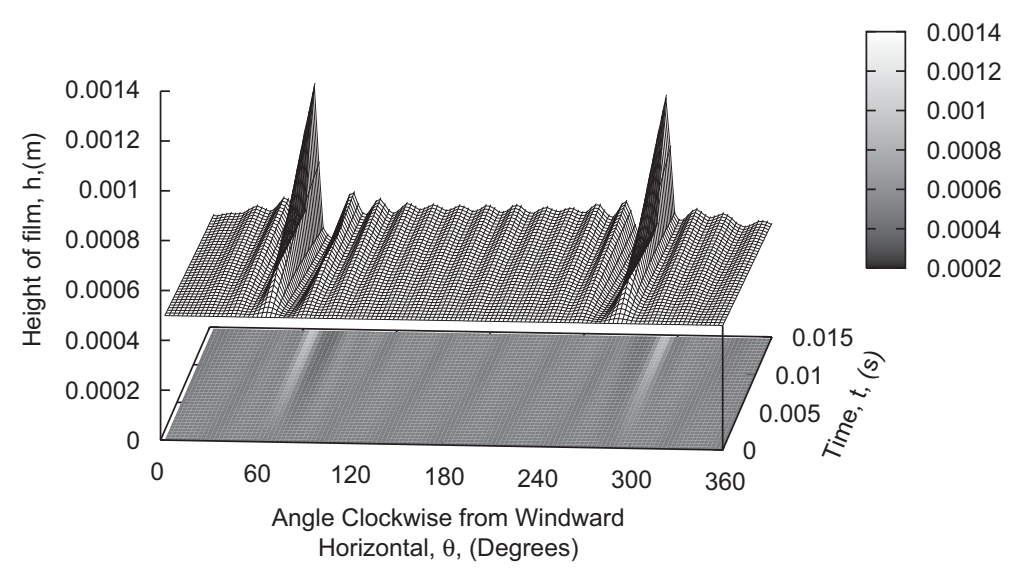

Fig. 16. Numerical prediction of the temporal evolution of film height in real time, under pressure and surface-tension effects only.

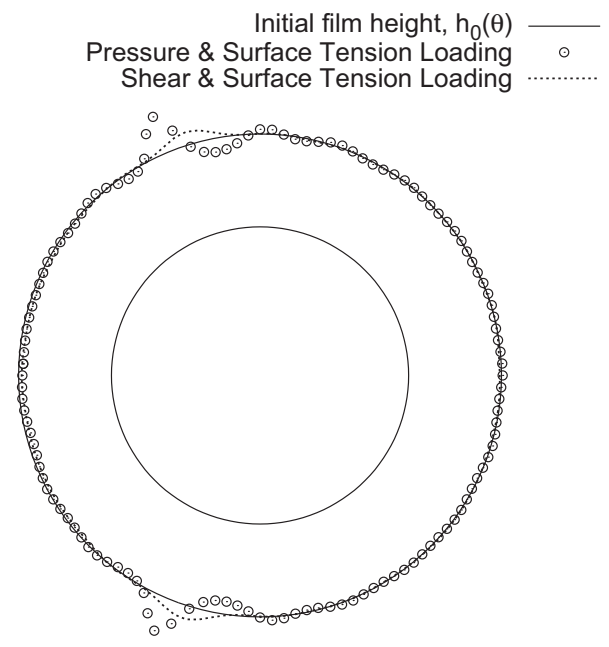

Fig. 17. Comparison of film height $(100 \times$ actual $)$ of present results under shear and surface-tension loading with those under pressure and surface-tension loading at $t=6.9 \times 10^{-3} \mathrm{~s}$, where the incident wind acts from the left.

attributed to the difference in the distribution of the aerodynamic coefficients, a point that will be discussed in depth in the following Section 5.3.1.

For consistency the present results were again compared with those of Lemaitre et al. (2007) under the same loading conditions; as Fig. 18 shows, the agreement is again excellent. As with the previous case the minor differences between the two solutions can again be attributed to differences in the distribution used for $C_{P}$ in the two schemes, since when the present solver was used to examine both forms of the governing evolution equation for the same distribution of $C_{P}$, the results were identical over the entire time range under investigation.

\subsubsection{Effect of pressure distribution}

To complement the work of Section 5.2.1, where the effect of differing magnitudes of applied shear loading but constant aerodynamic coefficient distribution was investigated, this section will focus on a study undertaken to examine the opposite situation, namely, how varying the distribution of the aerodynamic coefficient affects the evolution of the film profile. To achieve this the original distribution of aerodynamic coefficient determined from Achenbach (1968), $C_{P}$ Achenbach, was compared to the distribution of $C_{P}$, for a Reynolds number of $1 \times 10^{5}$, given by the formulae in ESDU (1986) Data Item (80025) which are an empirical fit based on numerous experiments over a range of Re, henceforth

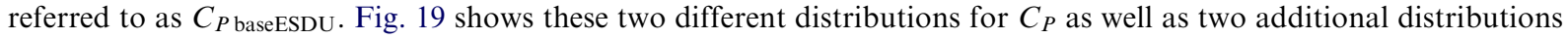
that were created such that the differences between $C_{P}$ Achenbach and $C_{P \text { baseESDU }}$ could be investigated. The definitions 


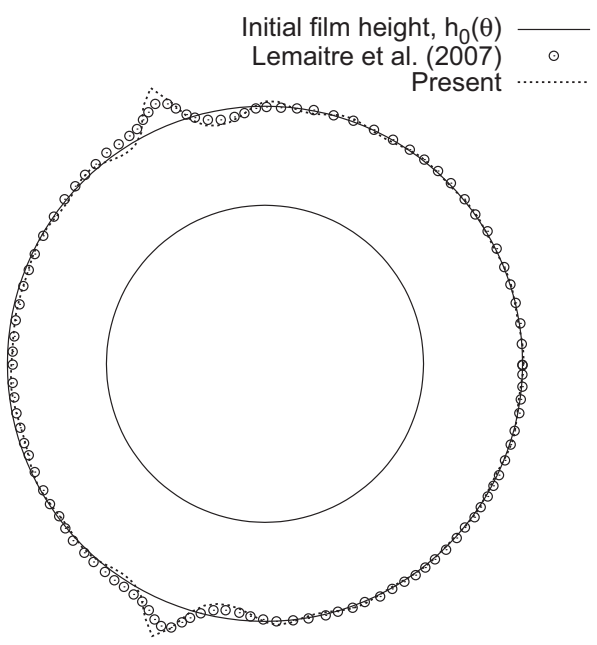

Fig. 18. Comparison of present numerical results with result of Lemaitre et al. (2007) for variation of film height $(100 \times$ actual $)$ at $t=6.9 \times 10^{-3} \mathrm{~s}$, under the effects of pressure and surface tension, where the incident wind acts from the left.

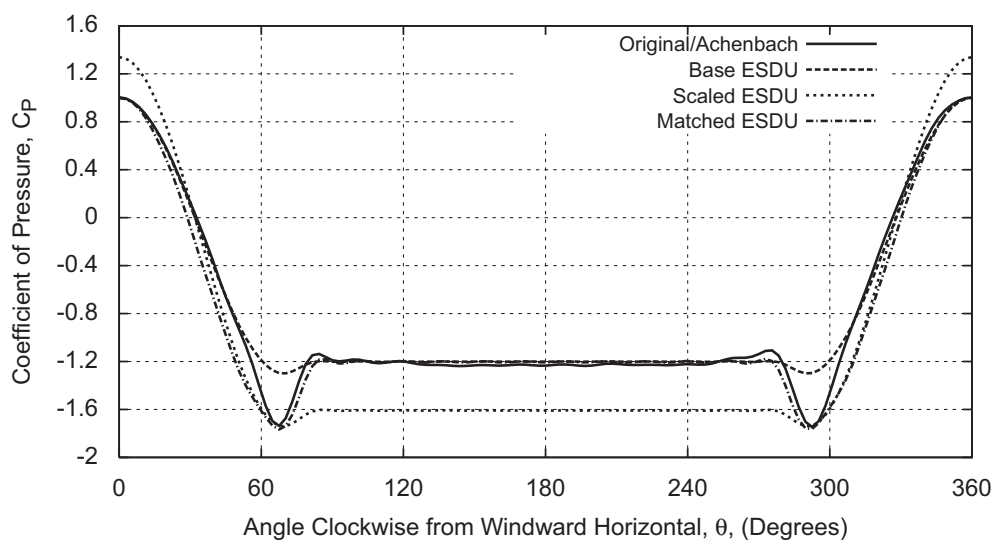

Fig. 19. Profiles of the four distributions of $C_{P}$ used, namely that used by Achenbach (1968), base ESDU, scaled ESDU and matched ESDU.

for these modified aerodynamic coefficient distributions, which were based upon $C_{P \text { baseESDU, and the reasons for }}$ choosing these, are as follows:

(i) Scaled ESDU-This ensured that the 'maximum' value of $C_{P}$ (in this case a minimum at peaks $\theta \simeq 75^{\circ}$ and $\theta \simeq 285^{\circ}$ ) matched that of Achenbach (1968) but in such a way that the same profile as the base ESDU distribution was maintained:

$$
C_{P \text { ScaledESDU }}=\frac{\left|\max C_{P \text { Achenbach }}\right|}{\left|\max C_{P \text { baseESDU }}\right|} \times C_{P \text { baseESDU }}
$$

This distribution of $C_{P}$ was chosen because it provided an additional case of the type studied in Section 5.2.1 and the means to examine whether it is the maximum value contained within, or the profile of, the aerodynamic coefficient distribution $C_{P}$ that has the greater effect on the evolutionary response.

(ii) Matched ESDU-This also ensured that the 'maximum' value of $C_{P}$ matched that of Achenbach (1968). Here, however, rather than 'scaling' the distribution, the equations for $C_{P \text { baseESDU }}$ were used as were, but with an artificially low minimum pressure coefficient $C_{P M}=-1.75$, which matched that of $C_{P}$ Achenbach. As a result, 


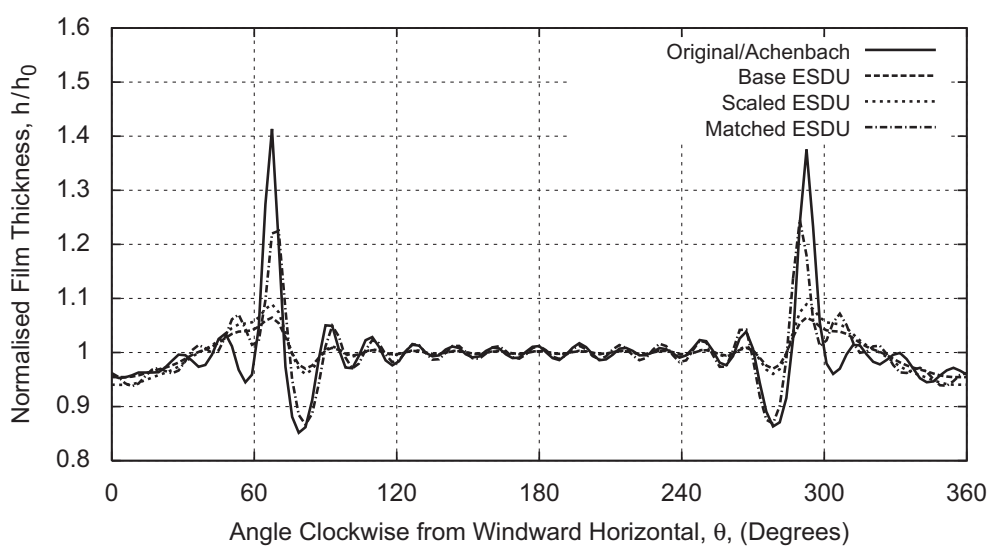

Fig. 20. Comparison of the effect that various distributions of $C_{P}$ have on the normalised film height profile, at $t=6.9 \times 10^{-3} \mathrm{~s}$, under pressure and surface-tension loading.

$C_{P \text { MatchedESDU }}$ gives a closer representation of $C_{P}$ Achenbach based on the ESDU formulae, as the frequency contents of the two distributions are closer. This distribution of $C_{P}$ was chosen as it provided a means of examining how varying the magnitudes of the coefficients $a_{k}$ and $b_{k}$ in the truncated Fourier series (16) and hence the frequency content affected the evolutionary response.

The results for normalised film thickness for the various pressure distributions $C_{P}$ at a given time, in this instance chosen to correspond to previous sections at $t=6.9 \times 10^{-3} \mathrm{~s}$, are shown in Fig. 20, and demonstrate that three distinct differences can be distinguished in the evolutionary response profiles.

First, due to the large variations in the value of $C_{P}$ over a small range of $\theta$ that accompanies the onset of adverse pressure gradient leeward of the separation points at $\theta \simeq 75^{\circ}$ and $\theta \simeq 285^{\circ}$ in the Achenbach and Matched ESDU distributions, and the faithful representation of actual experimental data in the former case, the higher frequency components of the truncated Fourier series have significantly greater magnitudes than those of the base ESDU and scaled ESDU distributions. This is reflected in increased magnitudes of coefficients $a_{k}$ and $b_{k}$ for large values of $k$ in the Achenbach and Matched ESDU distributions. As a result of these higher frequency components the profiles of evolutionary film thickness $h$ for the Achenbach and Matched ESDU distributions of $C_{P}$ display a noticeable wavelength, which corresponds to the highest Fourier component considered in the truncated series, and is therefore a numerical feature rather than a fundamental property. However, the effect of this feature is greatly magnified by the normalisation in Fig. 20, and is not noticeable in Fig. 18, which also presents the film thickness for the Achenbach distribution (at 100 times actual thickness), where the dominant feature is the rivulet located at approximately the separation points on the dry cylinder. Fig. 20 also shows that such fluctuations also occur in the profiles of evolutionary film thickness based on the base ESDU and scaled ESDU distributions, but that these are much less noticeable and the profiles of film thickness are considerably smoother.

Secondly, although a rivulet can be detected with all four distributions of $C_{P}$ at approximately the separation points of the dry cylinder, there is little correspondence between the heights or shapes of the rivulets. Those determined from $C_{P}$ Achenbach and $C_{P}$ MatchedESDU are considerably 'thicker' (i.e. $h / h_{0}$ is larger) and 'narrower' (i.e. small angular range of $\theta$ ), while those from $C_{P \text { baseESDU }}$ and $C_{P \text { ScaledESDU }}$ are 'thinner' (i.e. $h / h_{0}$ is smaller), 'wider' (i.e. large angular range of $\theta$ ) and considerably more asymmetric about the thickest point, in that these display a shallow increase in height windward of maximum thickness and a sharp decrease leewards. Furthermore although the maximum value of $C_{P}$ was the same in the $C_{P}$ Achenbach, $C_{P}$ MatchedESDU and $C_{P}$ ScaledESDU cases, the evolutions show that this has little bearing on the maximum height of the film; this point is further emphasised by the differences in the heights that result from the $C_{P}$ Achenbach and $C_{P}$ MatchedESDU distributions, since although these distributions have identical magnitudes and similar profiles, the differences in magnitudes of the frequency content of these coefficients, particularly in the upper frequency range, result in a rivulet of greater height forming in the $C_{P}$ Achenbach case. As such, the evolutionary responses with different distributions of $C_{P}$ produce different free surface profiles. Although not reported explicitly due to the absence of a suitable theoretical distribution of $C_{F}$, a corresponding result was likewise found under different distributions of shear loading, $C_{F}$.

Finally, as was true for the combination of shear and surface-tension loading, the present two cases of constant profile of $C_{P}$ distributions but varying magnitude ( $C_{P \text { baseESDU }}$ and $C_{P \text { ScaledESDU }}$ ) showed results that are once again 
scaled images of one another throughout the range studied. This again can be attributed to the dominance of the pressure loading over the surface-tension loading.

\subsubsection{Effect of varying Reynolds number}

Using the typical ranges of wind velocity $(\simeq 5-15 \mathrm{~m} / \mathrm{s})$ and cable diameter $(100-250 \mathrm{~mm})$ given previously, a range of Reynolds number over which RWIV could occur can be determined, namely $0.33 \times 10^{5}<\operatorname{Re}<2.5 \times 10^{5}$, which is significantly larger than that determined experimentally by Cosentino et al. (2003), namely $0.5 \times 10^{5}<\operatorname{Re}<1.5 \times 10^{5}$. In the latter range of $\mathrm{Re}$, however, the distribution of the pressure coefficient $C_{P}$ determined from the formulae given in ESDU 80025 is almost independent of Re, assuming the form of $C_{P \text { baseEsDu }}$ used in Section 5.3.1. As such if a constant profile of aerodynamic loading is assumed and only the velocity is altered then the results at differing Reynolds numbers are again found to be scale images of each other at any specific instant in time, as was found to be the case for $C_{F}$ (Section 5.2.1) and $C_{P}$ (Section 5.3.1).

Turbulence of the incoming flow, surface roughness and changes in the thin film geometry could, however, all cause a significant reduction in the critical Reynolds number and thus the flow itself may not always occur in the sub-critical flow regime as previously assumed. To investigate what effect such a change in flow regime would have upon the evolutionary response at a given flow speed, here chosen as $V=11.0 \mathrm{~m} / \mathrm{s}$, two additional distributions of $C_{P}$ and $C_{F}$ from different flow regimes were considered. These were again based upon experimental data obtained by Achenbach (1968) and were taken at a Reynolds number just below critical of $2.4 \times 10^{5}$, and at a super-critical $3.6 \times 10^{6}$. Likewise the aerodynamic coefficients were again represented by a twenty-term truncated Fourier series, Eq. (16). The results show that for both the shear, Fig. 21, and the pressure-loading cases, Fig. 22, a variation in flow regime resulted in a variation in rivulet location and size, with the flow regime corresponding to a larger Reynolds number typically resulting in a 'thinner' rivulet forming at a greater angle from the incident flow, the location of which was in good agreement with the positions of the minimum values of the aerodynamic coefficient distributions in all cases. Should a symmetric distribution of $C_{P}$ or $C_{F}$ be used, then the resulting evolutionary profile and rivulets are again found to be symmetric with respect to the incident flow. However, due to the faithful reproduction of slight asymmetries in the experimental data, the evolutionary profiles shown in Figs. 21 and 22 produced here are once again slightly asymmetric, with the rivulet on the lower surface in the super-critical case displaying the largest deviation.

In practice, a variation in Reynolds number is likely to be associated with a change in both the incident wind speed and the aerodynamic distribution, although this will likely not be as large as shown here unless it results in a shift in flow regime. Therefore, the two effects outlined here would probably act in union, the extent of each contribution depending upon the particular case under examination. What is clear, however, is that in all cases and in under both the sub-critical and super-critical flow regimes rivulets were again found to form on both the upper and lower surfaces marginally upstream of the expected separation point of a dry cylinder at the same Reynolds number.

\subsection{Full loading}

The temporal evolution of film thickness for all four loading conditions (pressure, shear, surface tension and gravity) acting simultaneously is shown in Fig. 23. Similarly to the previous two cases which examined the effect of combinations of shear or pressure with surface-tension loading (Sections 5.2 and 5.3, respectively), under full loading conditions two distinct rivulets can be seen to form. However, in this instance the symmetry about the axis of the incident wind of the previous cases, should a symmetric profile be used, is lost due to the effect of gravity. This gravitational loading results in the rivulets that evolve under full loading conditions being thicker on the lower surface and thinner on the upper surface than those examined previously. This can be quantified by means of the normalised height of the rivulet $h / h_{0}$ at the specific time instant used for comparisons in the previous cases of $t=6.9 \times 10^{-3} \mathrm{~s}$. For the upper rivulet this ratio is $h_{\text {upper }} / h_{0}=1.38$ and for the lower rivulet it is $h_{\text {lower }} / h_{0}=1.67$, where $h_{\text {upper }}$ and $h_{\text {lower }}$ are the heights of the upper and lower rivulet at the point of maximum thickness, respectively, which is not fixed in $\theta$. Fig. 24 demonstrates this temporal increase in height of both rivulets while also highlighting that the lower rivulet grows faster than the upper rivulet due to the effect of gravity, as would be expected. The latter is achieved by plotting the ratio between the film thickness of the lower and upper rivulets $\left(h_{\text {lower }} / h_{\text {upper }}\right)$. Furthermore, while the point of maximum thickness of the lower rivulet moves leeward from the point where it occurred under only pressure and surface-tension loading (Section 5.3), specifically from $\theta \simeq 288^{\circ}$ to $\theta \simeq 282^{\circ}$, the thinner upper rivulet moves windward from $\theta \simeq 66^{\circ}$ to $\theta \simeq 59^{\circ}$. This simple result agrees with intuitive expectations as to the effects of gravity on the system, and in so doing increases confidence in the modelling approach. 


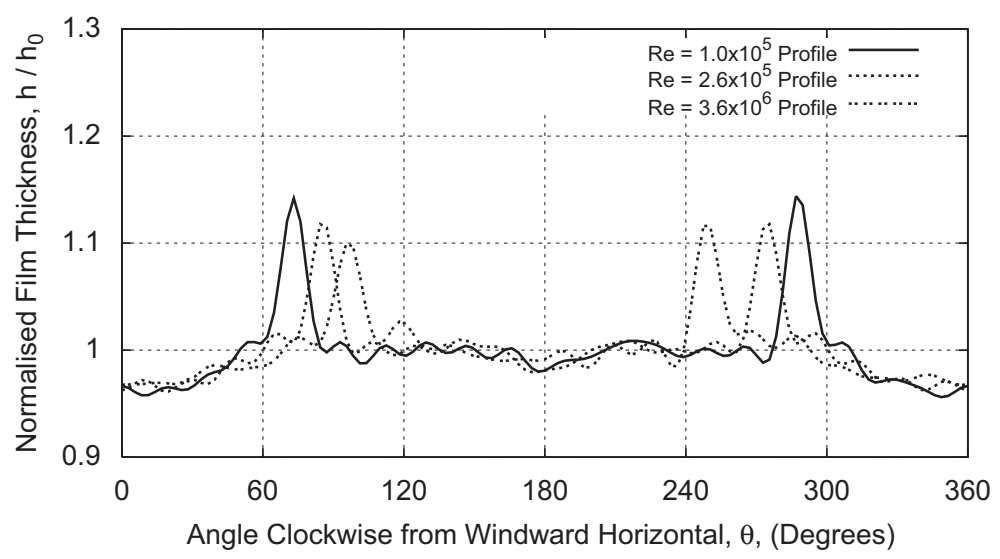

Fig. 21. Effect of varying Reynolds number through change of flow regime on the variation of normalised film height with angle from windward horizontal at $t=6.9 \times 10^{-3} \mathrm{~s}$, for shear and surface-tension loading.

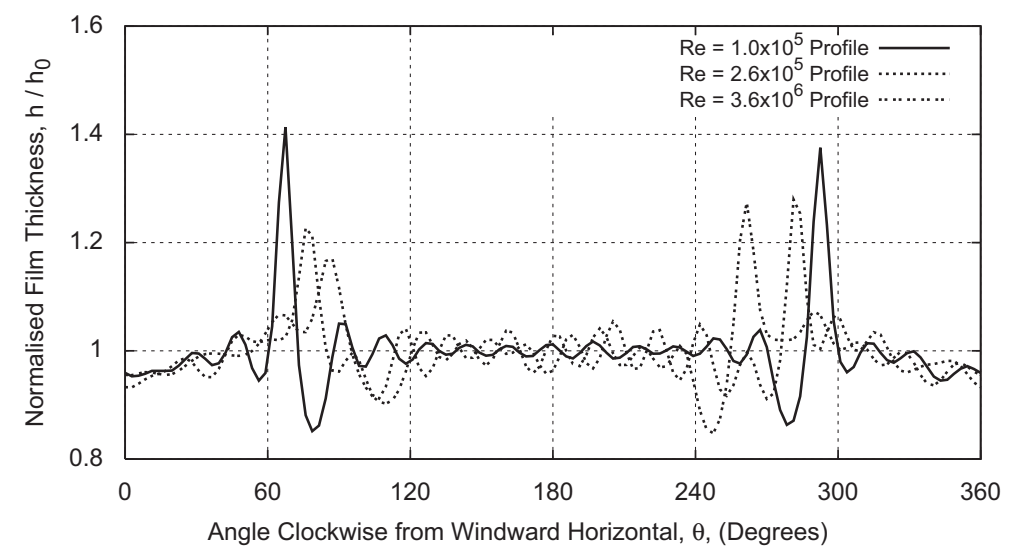

Fig. 22. Effect of varying Reynolds number through change of flow regime on the variation of normalised film height with angle from windward horizontal at $t=6.9 \times 10^{-3} \mathrm{~s}$, for pressure and surface-tension loading.

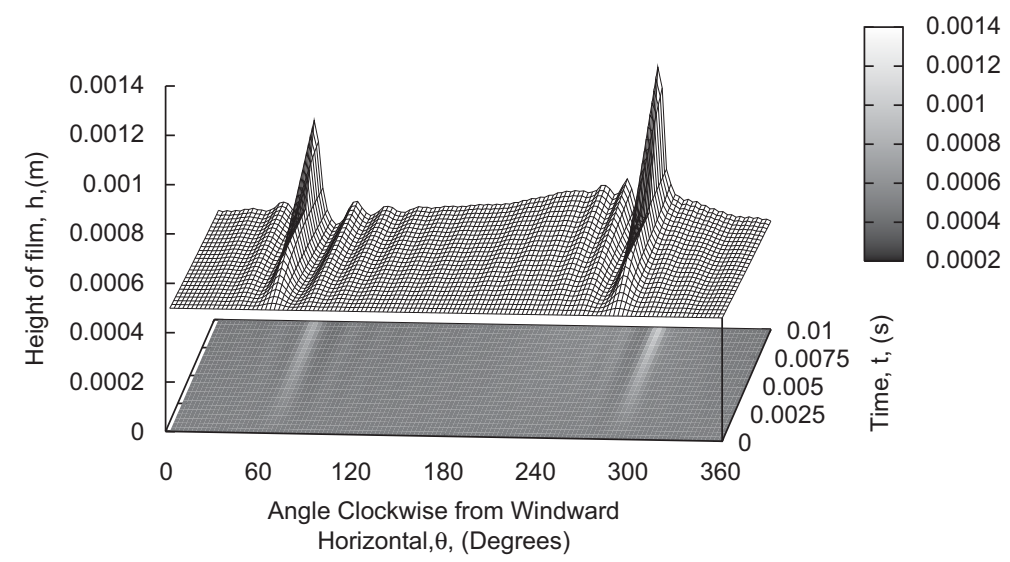

Fig. 23. Numerical prediction of temporal evolution in real time of film height under full loading conditions. 


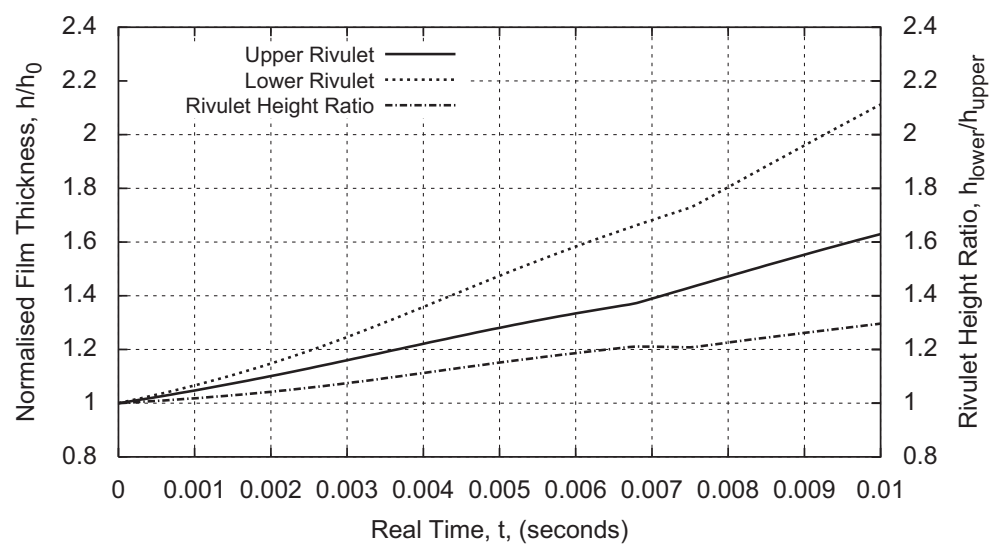

Fig. 24. Early temporal evolution of normalised film height of upper and lower rivulets and ratio between these.

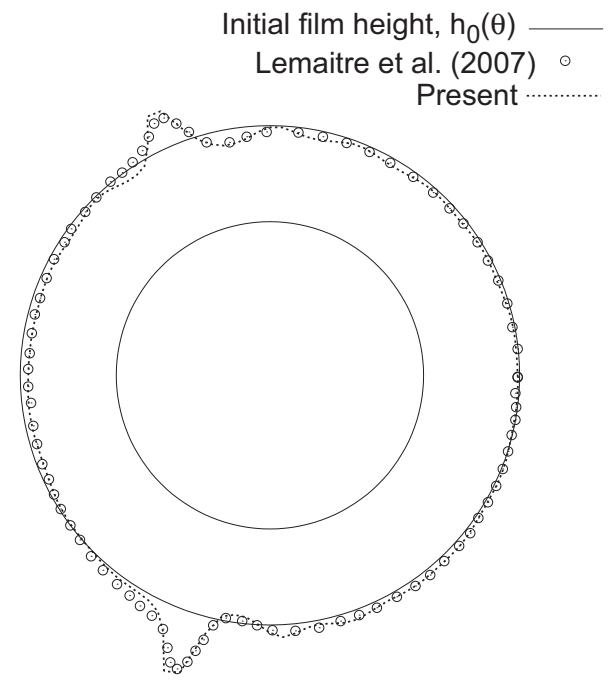

Fig. 25. Comparison of present numerical results with result of Lemaitre et al. (2007) for variation of film height (100 $\times$ actual) at $t=6.9 \times 10^{-3}$ s under full loading conditions, where the incident wind acts from the left.

For consistency these results were again compared with those of Lemaitre et al. (2007) under the same loading conditions; as Fig. 25 shows, the agreement is again excellent. As with the previous cases, the minor differences between the two solutions can be attributed to a variation in the distribution used for $C_{P}$ and $C_{F}$ in the two schemes, since when the present solver was used to examine both forms of the governing evolution equation for the same distribution of $C_{P}$ and $C_{F}$ (in particular, those determined in Section 3.1) the results were identical over the entire time range under investigation. Although a comparison has been made with the work of Lemaitre et al. (2007) for each of the loading combinations presently examined, due to the complexity of the distributions of aerodynamic coefficient used, analytical results are not available to verify against and hence the present data has been verified against the only other computational data available. As the two numerical solutions have been obtained independently and the results have been generated by two separate solvers, it allows a greater degree of confidence in both sets of results and the method used. That said, the present work also highlights several new features for the problems investigated, notably presenting both the temporal evolution of the rivulet and profiles at specific time instants, and examining how the magnitude of loading, distributions of aerodynamic coefficients and Reynolds number affect the evolutionary response.

Finally, Fig. 26 displays the evolutionary profiles from the present solver for the rivulets formed under the three loading cases, shear and surface tension, pressure and surface tension, and full loading at $t=6.9 \times 10^{-3} \mathrm{~s}$. This 


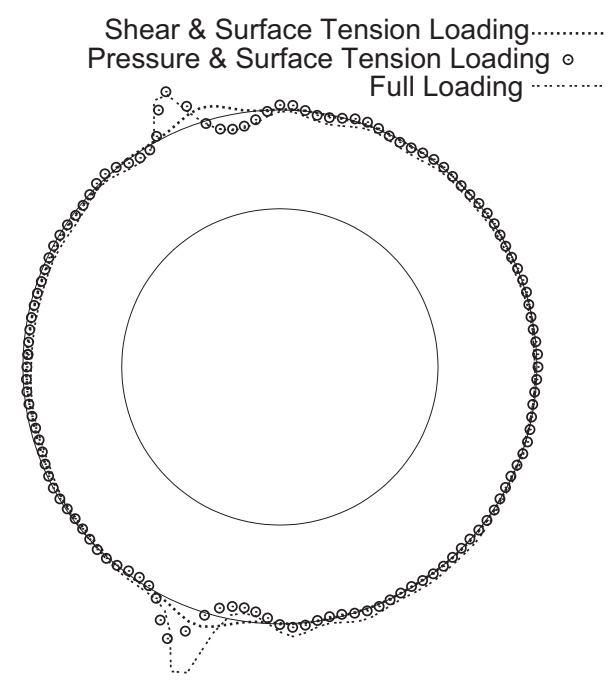

Fig. 26. Comparison of present numerical results under three loading conditions for variation of film height $(100 \times$ actual $)$ at $t=6.9 \times 10^{-3} \mathrm{~s}$, where the incident wind acts from the left.

highlights, in a single figure, the differences in form and location of the rivulets discussed in the previous sections, with the rivulets forming under shear loading being 'wider' and 'thinner' than those from pressure loading while forming at greater angles from the incident flow, albeit in both cases still at approximately the separation points of the dry cylinder. Furthermore, the slight asymmetries in these results, which result from those of the aerodynamic coefficients, are put into context when compared to the effect of gravity, which truly results in an asymmetric film height distribution about the incident flow, resulting in a windward movement of the upper rivulet and a leeward movement and thickening of the lower rivulet.

Overall, therefore, by describing the formation and evolution of rivulets in the approximate locations highlighted by previous studies (Hikami and Shiraishi, 1988; Gu and Du, 2005; Matsumoto et al., 2007) as critical to the RWIV mechanism, the present study establishes that conditions required by proposed mechanisms, such as galloping (Matsumoto et al., 2007), could indeed occur due to the presence of a thin film of fluid on the outer surface of a cylinder in an airflow. The confirmation of this result is therefore significant in addressing the overall RWIV problem. The next step, namely considering the coupling of the present numerical method with an aerodynamic solver should hopefully further this process in addition to investigating what role the temporal evolution of geometry has on the overall system response.

\section{Conclusions}

A numerical model for the evolution of a thin film of liquid on the outer surface of a stationary circular cylinder under the combined effects of pressure, shear, surface tension and gravity has been presented. The pseudo-spectral solver developed to solve the governing evolution equation numerically has been demonstrated to be both selfconsistent and to show excellent agreement with previous analytical and computational investigations, for all the verification studies undertaken.

For the shear and surface-tension loading, and the pressure and surface-tension loading cases, two symmetric rivulets were found to form just windward of the separation points of a dry cylinder, and a comparison between these two loadings revealed that they have a similar effect on the film-thickness response. Under full loading conditions, although two rivulets were again found to form, these were asymmetric about the horizontal axis of incident wind and of different height, due to the effect of gravity.

Through variation of the magnitude of the applied shear or pressure loading, with a constant distribution of aerodynamic coefficient $C_{F}$ or $C_{P}$ maintained, it has been shown that the evolutionary responses of film thickness retain the same profile but have different magnitude, and that this scaling is proportional to the magnitude of incident loading. This can be attributed to the dominance of the loading due to the external aerodynamic field over that of the surface tension for the range of parameters investigated. 
In contrast, changing the distribution of aerodynamic coefficients $C_{F}$ and $C_{P}$ markedly altered the evolutionary response of film thickness. Although for all the cases studied, two rivulets did form, the sizes and profiles of these were significantly different from one another. As a result the distribution of aerodynamic loading was determined to have a significantly greater effect on the evolution response of film thickness than the magnitude of loading for the cases examined.

Should a variation in Reynolds number result from simply a change in magnitude of incident velocity for a constant distribution of aerodynamic coefficient then the profile of film thickness again results in the formation of scale images. However, should the change in Re result in a varying distribution of $C_{F}$ or $C_{P}$, such as would happen with a change in flow regime, then the evolutionary profile shows a variation in both the location and the form of the rivulets, although in both the sub- and super-critical regimes rivulets were found to form marginally upstream of the separation points of a dry cylinder at the same Reynolds number. Capturing numerically the formation and evolution of rivulets in the approximate locations highlighted by previous studies is a significant result in the overall RWIV problem.

\section{Acknowledgement}

The first and fifth authors, A.C. Robertson and J.M. Sullivan, gratefully acknowledge the support of the UK Engineering and Physical Sciences Research Council (EPSRC) via Doctoral Training Account studentships.

\section{Appendix: Analytical solution for constant shear}

The evolution equation for the free surface profile $h(\theta, t)$ is

$$
h_{t}+\left[-\frac{1}{3 \mu R}\left(\rho g \cos \theta-\frac{\gamma}{R^{3}}\left(h+h_{\theta \theta}\right)_{\theta}+\frac{P_{\theta}}{R}\right) h^{3}+\frac{T h^{2}}{2 \mu R}\right]_{\theta}=0 .
$$

If surface tension, gravity and the external pressure gradient are neglected then this becomes

$$
h_{t}+\left(\frac{T h^{2}}{2 \mu R}\right)_{\theta}=0
$$

a first-order partial differential equation for $h(\theta, t)$. For simplicity we take $T$ to be a constant; then (A.2) becomes

$$
h_{t}+\frac{T}{\mu R} h h_{\theta}=0 .
$$

The characteristic equations associated with Eq. (A.3) are

$$
\frac{\mathrm{d} h}{\mathrm{~d} t}=0, \quad \frac{\mathrm{d} \theta}{\mathrm{d} t}=\frac{T h}{\mu R},
$$

whose solutions are

$$
h=\text { constant }, \quad \theta-\frac{T h}{\mu R} t=\text { constant. }
$$

Therefore, the general solution $h(\theta, t)$ of (A.3) satisfying the initial condition $h(\theta, 0)=h_{0}(\theta)$ (where $h_{0}(\cdot)$ is some prescribed non-negative function satisfying $\left.h_{0}(\theta+2 \pi)=h_{0}(\theta)\right)$ is given implicitly by

$$
h=h_{0}\left(\theta-\frac{T h}{\mu R} t\right) .
$$

For example, if $h_{0}(\theta)=H(1-a \cos (n \theta))$ (with $|a|<1, n=1,2,3, \ldots$ ) then (A.6) gives the implicit equation

$$
\frac{h}{H}=1-a \cos \left[n\left(\theta-\frac{T h}{\mu R} t\right)\right]
$$

determining $h$ as a function of $\theta$ and $t$.

We note that Eq. (A.6) predicts that $h$ stays non-negative for all $t$ and $\theta$ (as expected on physical grounds). Also if $h_{0}=0$ at some station $\theta=\theta_{0}$ then $h\left(\theta_{0}, t\right)=0$ for all $t$, that is, any 'three-phase contact line' does not move. 
The solution (A.6) will become invalid if the wavelike profile of the free surface 'breaks' at some instant, that is, if $\partial h / \partial \theta$ becomes infinite. Differentiating (A.6) we obtain

$$
\frac{\partial h}{\partial \theta}=\frac{h_{0}^{\prime}(\xi)}{1+h_{0}^{\prime}(\xi) \frac{T}{\mu R} t},
$$

where

$$
\xi=\theta-\frac{T h}{\mu R} t
$$

Thus the free surface will 'break' at the instant when $1+h_{0}^{\prime}(\xi) T t / \mu R$ first takes the value zero, that is, at the instant $t=t_{\mathrm{s}}$ defined by

$$
t_{\mathrm{s}}=\frac{\mu R}{T} \min _{\xi}\left(-\frac{1}{h_{0}^{\prime}(\xi)}\right)
$$

provided that $t_{\mathrm{s}}$ is positive. If $\xi=\xi_{\mathrm{s}}$ is the value of $\xi$ at which this minimum occurs then by (A.6) and (A.9) the shock occurs at height $h=h_{0}\left(\xi_{\mathrm{s}}\right)\left(=h_{\mathrm{s}}\right.$, say) at position $\theta=\theta_{\mathrm{s}}$ given by

$$
\theta_{\mathrm{s}}=\xi_{\mathrm{s}}+\frac{T h_{\mathrm{s}}}{\mu R} t_{\mathrm{s}}(\bmod 2 \pi) \text {. }
$$

For example, if $h_{0}(\theta)=H(1-a \cos (n \theta))$ (with $\left.|a|<1, n=1,2,3, \ldots\right)$, then $h_{0}^{\prime}(\xi)=n H a \sin (n \xi)$, so that (A.10) gives

$$
t_{\mathrm{s}}=\frac{\mu R}{n H a T} \min _{\xi}\left(-\frac{1}{\sin (n \xi)}\right) \quad\left(t_{\mathrm{s}}>0\right) ;
$$

therefore

$$
\xi_{\mathrm{s}}=\frac{3 \pi}{2 n}, \quad t_{\mathrm{s}}=\frac{\mu R}{n H a T}, \quad h_{\mathrm{s}}=H, \quad \theta_{\mathrm{s}}=\frac{3 \pi}{2 n}+\frac{1}{n a}(\bmod 2 \pi) .
$$

\section{References}

Achenbach, E., 1968. Distribution of local pressure and skin friction around a circular cylinder in a cross flow up to Re $=5 \times 10^{6}$. Journal of Fluid Mechanics 34, 625-639.

Bosdogianni, A., Olivari, D., 1996. Wind and rain induced oscillations of cables of stayed bridges. Journal of Wind Engineering and Industrial Aerodynamics 64, 171-185.

Cosentino, N., Flamand, O., Ceccoli, C., 2003. Rain-wind induced vibration of inclined stay cables. Part 1: experimental investigation and physical explanation. Wind and Structures 6, 471-484.

ESDU, 1986. Mean forces, pressures and flow field velocities for circular cylindrical structures: single cylinder with two-dimensional flow. Engineering Sciences Data Unit International, Item 80025, Amendment C.

Flamand, O., 1995. Rain-wind induced vibration of cables. Journal of Wind Engineering and Industrial Aerodynamics 57, $353-362$.

Fornberg, B., 1996. A Practical Guide to Pseudospectral Methods. Cambridge University Press, Cambridge.

Geurts, C., Vrouweuvelder, T., van Staalduinen, P., Reusink, J., 1998. Numerical modelling of rain-wind induced vibration: Erasmus Bridge, Rotterdam. Structural Engineering International 8, 129-135.

Gu, M., Du, X., 2005. Experimental investigation of rain-wind-induced vibration of cables in cable-stayed bridges and its mitigation. Journal of Wind Engineering and Industrial Aerodynamics 93, 79-95.

Gu, M., Huang, L., 2008. Theoretical and experimental studies on the aerodynamic instability of a two-dimensional circular cylinder with a moving attachment. Journal of Fluids and Structures 24, 200-211.

Hikami, Y., Shiraishi, N., 1988. Rain-wind induced vibration of cables in cable stayed bridges. Journal of Wind Engineering and Industrial Aerodynamics 29, 409-418.

Lemaitre, C., Hémon, P., de Langre, E., 2007. Thin water film around a cable subject to wind. Journal of Wind Engineering and Industrial Aerodynamics 95, 1259-1271.

Matsumoto, M., Saitoh, T., Kitazawa, M., Shirato, H., Nishizaki, T., 1995. Response characteristics of rain-wind induced vibration of stay-cables of cable-stayed bridges. Journal of Wind Engineering and Industrial Aerodynamics 57, 323-333.

Matsumoto, M., Yagi, T., Adachi, Y., Hatsuda, H., Shima, T., 2007. Karman vortex effects on aerodynamic instabilities of inclined stay-cables. In: Proceedings of the 12th International Conference on Wind Engineering, Cairns, Australia, pp. $175-182$.

Ni, Y.Q., Wang, X.Y., Chen, Z.Q., Ko, J.M., 2007. Field observations of rain-wind-induced cable vibration in cable-stayed Dongting Lake Bridge. Journal of Wind Engineering and Industrial Aerodynamics 95, 303-328. 
Peil, U., Dreyer, O., 2007. Rain-wind induced vibrations of cables in laminar and turbulent flow. Wind and Structures $10,83-97$.

Reisfeld, B., Bankoff, S.G., 1992. Non-isothermal flow of a liquid film on a horizontal cylinder. Journal of Fluid Mechanics 236, 167-196.

Robertson, A.C., Taylor, I.J., 2007. Effect of rivulets on a circular cylinder using a 2D discrete vortex method. In: Proceedings of the 12th International Conference on Wind Engineering, Cairns, Australia, pp. 863-870.

Verwiebe, C., Ruscheweyh, H., 1998. Recent research results concerning the exciting mechanisms of rain-wind-induced vibrations. Journal of Wind Engineering and Industrial Aerodynamics 74-76, 1005-1013.

Wang, Z.J., Zhou, Y., Huang, J.F., Xu, Y.L., 2005. Fluid dynamics around an inclined cylinder with running water rivulets. Journal of Fluids and Structures 21, 49-64.

Yamaguchi, H., 1990. Analytical study on the growth mechanism of rain vibration of cables. Journal of Wind Engineering and Industrial Aerodynamics 33, 78-80.

Zuo, D., Jones, N.P., Main, J.A., 2008. Field observation of vortex- and rain-wind-induced stay-cable vibrations in a threedimensional environment. Journal of Wind Engineering and Industrial Aerodynamics 96, 1124-1133. 